\title{
Core Turbulent transport in tokamak plasmas: bridging theory and experiment with QuaLiKiz
}

\author{
C. Bourdelle ${ }^{1}$, J. Citrin ${ }^{1,2}$, B. Baiocchi ${ }^{3}$, A. Casati ${ }^{1}$, P. Cottier ${ }^{1}$, X. Garbet ${ }^{1}$, F. Imbeaux ${ }^{1}$, JET Contributors* \\ ${ }^{1}$ CEA, IRFM, F-13108 Saint-Paul-lez-Durance, France. \\ ${ }^{2}$ FOM Institute DIFFER - Dutch Institute for Fundamental Energy Research, \\ P.O. box 6336, $5600 \mathrm{HH}$ Eindhoven, The Netherlands \\ ${ }^{3}$ Istituto di Fisica del Plasma CNR-EURATOM, via Cozzi 5320125 Milano, Italy and \\ * EUROfusion Consortium, JET, Culham Science Centre, Abingdon, \\ OX14 3DB, UK, See the Appendix of F. Romanelli et al., \\ Proceedings of the 25th IAEA Fusion Energy Conference 2014, Saint Petersburg, Russia
}

\begin{abstract}
Nonlinear gyrokinetic codes allow for detailed understanding of tokamak core turbulent transport. However, their computational demand precludes their use for predictive profile modeling. An alternative approach is required to bridge the gap between theoretical understanding and prediction of experiments. A quasilinear gyrokinetic model, QuaLiKiz [C. Bourdelle, X. Garbet, et al, Phys. Plasmas 14, 112501 (2007)], is demonstrated to be rapid enough to ease systematic interface with experiments. The derivation and approximation of this approach are reviewed. The quasilinear approximation is proven valid over a wide range of core plasma parameters. Example of profile prediction using QuaLiKiz coupled to the CRONOS integrated modeling code [J.F. Artaud et al Nucl. Fusion, 50043001 (2010)] are presented. QuaLiKiz is being coupled to other integrated modeling platforms such as ETS and JETTO. QuaLiKiz gyrokinetic quasilinear turbulent fluxes are available to all users. It allows for extensive stand-alone interpretative analysis and for first principle based integrated predictive modeling.
\end{abstract}

\section{INTRODUCTION}

Understanding and predicting temperature, density and rotation profiles in the confined core of tokamak plasmas requires accurate and rapid turbulent transport codes.

The continuously expanding availability of high performance computing systems facilitates the use of gyrokinetic codes, either gradient driven such as GENE [1], GKW [2], GS2 [3], and GYRO [4] or flux driven such as GYSELA [5] or GT5D [6]. Fixed gradient codes were intensively applied over the past few years for quasilinear and nonlinear investigations of experimental profiles. The role of the particle convection in determining the density profile was identified and understood [7-9]. The impurity transport level was successfully compared to quasilinear diffusive and convective coefficients [1013], including W turbulent transport [14-16]. Angular momentum convective transport was also explored [17-19].

Despite impressive progress in comparing quasilinear gyrokinetic and measured fluxes, the main issue - predicting temperature, density, rotation profiles in future tokamaks such as ITER - remains highly challenging. This requires the dynamic solution of the transport equations. Beyond the fluxes, this includes energy, particle, and momentum source modeling, together with a self-consistent magnetic equilibrium. This system is characterized by numerous nonlinear interactions, e.g., between temperature and current diffusion, and density and radio frequency heating. To reach a stationary state, these interactions must be modeled for a few confinement times, i.e. $\sim 1 \mathrm{~s}$ for JET-scale plasmas. This necessitates approximately 10000 flux calculations. A severe constraint on the transport model calculation time per call of a few minutes is set to obtain the profiles evolution over $1 \mathrm{~s}$ of plasma within reasonable wall-clock times (e.g. $<24 \mathrm{~h})$ and on an very easily accessible number of computing cores (e.g. $\simeq 30$ ). Gradient driven gyrokinetic nonlinear simulations are too costly, taking typically 5000 to 50000 CPU hours per flux calculation for a single radial position. While optimized multiscale coupling schemes do exist [20], routine profile calculations and sensitivity studies are still impractical. Therefore, the quasilinear approximation provides an avenue for tractable profile calculations. Table I situates quasilinear codes with respect to the more complete gyrokinetic nonlinear codes.

As summarized in table I, the quasilinear approximation alone is not sufficient to reach the required level of tractability. Indeed, the quasilinear approximation alone provides only a factor 100 speedup compared with nonlinear. Hence, further approximations are necessary. In TGLF [23, 24], the fluid instead of the gyrokinetic dispersion relation is solved. In QuaLiKiz [8], the axisymmetric gyrokinetic problem is further simplified thanks to the use of the lowest order ballooning transform. Some integrals are reduced by treating separately trapped and passing particles. Finally, in QuaLiKiz, the eigenfunctions are not solved self-consistently together with the eigenvalues, but rather are calculated in the fluid limit. See table II for a general overview of QuaLiKiz and TGLF respective frameworks. Previously to TGLF, other fluid codes have been developed and widely used such as the Weiland model [25] (embedded in the MMM model [26, 27]), the IFS-PPPL model [21] and GLF23 [28] now extended in TGLF. TGLF has been extensively tested and its predicted profiles of electron and ion temperature have been shown to reproduce measured profiles from the core to $\rho=0.8$ with an averaged RMS error around 15\% [29]. Hybrid scenarios [30] and ITB were also modeled [31]. QuaLiKiz has also been extensively compared to nonlinear gyrokinetic codes and to TGLF itself as summarized in table III later in this paper. Despite these successes, numerous issues remain to be addressed, such as: the pedestal height prediction [32], the underestimated transport level in the deep core, the transport level in the L mode edge region [33], the combined role of MHD and turbulence on the confinement, the impact of finite $\beta$ in particular in hybrid scenarios [34], etc. Nonetheless, the overall success of quasilin- 


\begin{tabular}{|c|c|c|c|}
\hline \multicolumn{4}{|c|}{$\rightarrow$ Towards easing interface with experiments $\rightarrow$} \\
\hline flux driven & \multicolumn{3}{|c|}{ gradient driven } \\
\hline $\begin{array}{l}\text { Gyrokinetic } \\
\text { full-f }\end{array}$ & \multicolumn{2}{|c|}{ Gyrokinetic $\delta f$} & $\begin{array}{l}\text { Gyro- } \\
\text { Landau } \\
\text { fluid }\end{array}$ \\
\hline $\begin{array}{l}\text { Some ex- } \\
\text { amples are: } \\
\text { GT5D [6], } \\
\text { GYSELA } \\
\text { [5] }\end{array}$ & 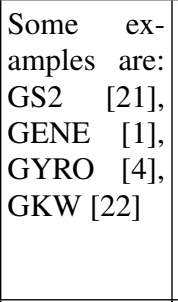 & \begin{tabular}{|l} 
Additional \\
approxi- \\
mations: \\
electro- \\
static, \\
$s-\alpha$, etc: \\
QuaLiKiz \\
{$[8]$}
\end{tabular} & $\begin{array}{l}\text { Calibrated } \\
\text { to gyroki- } \\
\text { netic theory } \\
\text { TGLF [23] }\end{array}$ \\
\hline Nonlinear & $\begin{array}{l}\text { Nonlinear or } \\
\text { quasilinear }\end{array}$ & \multicolumn{2}{|l|}{ Quasilinear } \\
\hline $\begin{array}{l}\text { Estimated } \\
\text { time to } \\
\text { simulate } 20 \\
\text { radial points } \\
\text { and } 10 \text { wave } \\
\text { numbers: } \\
\text { around } 10 \\
\text { Mh }\end{array}$ & $\begin{array}{l}\text { Around } 50 \\
000 \text { h for } \\
\text { nonlinear } \\
\text { runs } \quad \text { or } \\
500 \text { h for } \\
\text { quasilinear } \\
\text { runs }\end{array}$ & \multicolumn{2}{|c|}{ Around 5 min. } \\
\hline
\end{tabular}

TABLE I: Various core turbulence modeling tools are presented from the codes dedicated to detailed theoretical investigation of fundamental turbulence mechanisms towards the codes able to provide turbulent fluxes estimates for systematic experimental comparison/prediction.

ear turbulent transport models in reproducing tokamak plasma profiles demonstrates the validity of the quasilinear approach in multiple regimes.

In QuaLiKiz, recent efforts in improving the numerical schemes have optimized the dispersion relation solver [35] and the plasma dispersion functions [36]. Thanks to these further approximations, 20 radial positions and 10 wave numbers are modeled within 5 minutes, meaning that, as TGLF, in an integrated framework, QuaLiKiz can model $1 \mathrm{~s}$ of plasma over 30 processors within $24 \mathrm{~h}$. QuaLiKiz is embedded in CRONOS [37], and was applied for predictive simulations of energy and particle transport for JET H-mode scenarios $[38,39]$. This article is focusing on QuaLiKiz development and results.

This paper aims at presenting QuaLiKiz in a brief and nonetheless exhaustive manner. References are provided throughout to allow the interested reader accessing the complete derivation, verification and validation of the code. Quasilinear fluxes, $\Gamma$, are sums over the wave number $k$ and the frequency $\omega$ made of two essential parts: the linear response $R_{l i n}(k, \omega)$ and the saturated potential $\left|\phi_{k \omega}\right|^{2}$; it can be expressed schematically as $\Gamma \propto \sum_{k} R_{\text {lin }}(k, \omega) \times\left|\phi_{k \omega}\right|^{2}$. Therefore this paper review the gyrokinetic linear dispersion relation leading to $R_{\text {lin }}(k, \omega)$ in section 2 , the rules and the validity of the saturated potential $\left|\phi_{k \omega}\right|^{2}$ in section 3 and finally, in section 4, the QuaLiKiz fluxes are compared to nonlinear gyrokinetic fluxes as well as experimentally measured profiles. Perspectives for further speed up while enlarging the validity domain are discussed in section 5 .

\begin{tabular}{|c|c|c|}
\hline & QuaLiKiz & TGLF \\
\hline Calculated fluxes & \multicolumn{2}{|c|}{$\begin{array}{l}\text { Energy, particle and momentum fluxes } \\
\text { for unlimited number of ions and elec- } \\
\text { trons }\end{array}$} \\
\hline Dispersion relation & 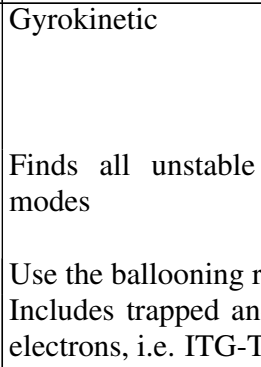 & $\begin{array}{l}\text { Fluid: } 12 \text { moments } \\
\text { for circulating par- } \\
\text { ticles and } 3 \text { for } \\
\text { trapped } \\
\text { Finds the top two } \\
\text { most unstable } \\
\text { modes } \\
\text { epresentation } \\
\text { d passing ions and } \\
\text { EM and ETG }\end{array}$ \\
\hline Eigenfunctions & $\begin{array}{l}\text { Estimated in the } \\
\text { fluid limit: shifted } \\
\text { Gaussians }\end{array}$ & $\begin{array}{ll}\text { Hermite } & \text { basis } \\
\text { functions, Gaussian } \\
\text { width to maximize } \\
\gamma \text { for } 1-2 \text { basis } \\
\text { functions } \\
\text { refined to } 4\end{array}$ \\
\hline Collisions & $\begin{array}{l}\text { On electrons, use } \\
\text { the Krook operator, } \\
\text { energy dependent }\end{array}$ & $\begin{array}{l}\text { On electrons, use } \\
\text { a pitch-angle scat- } \\
\text { tering operator, en- } \\
\text { ergy dependent }\end{array}$ \\
\hline Equilibrium & $s-\alpha$ & $\begin{array}{l}\text { Bishop eikonal } \\
\text { with Miller flux } \\
\text { surface r shape } \\
\text { with elongation, } \\
\text { triangularity [40] }\end{array}$ \\
\hline $\begin{array}{l}\text { Saturated potential: } \\
\text { level, } \mathrm{k} \text { and } \\
\text { frequency spectra }\end{array}$ & $\begin{array}{l}\text { Mixing length: } \\
\gamma /<k_{\perp}^{2}>\text { with } \\
k_{\perp} \text { accounting for } \\
\text { the eigenfunction } \\
k_{\theta} \text { spectrum such } \\
\text { that } k_{\theta}^{-3} \text { above the } \\
\text { maximum and } k_{\theta} \\
\text { below } \\
\text { Frequency spec- } \\
\text { trum: a Lorentzian } \\
\text { which width scales } \\
\text { as } \gamma \text { adjusted for } \\
|s|<0.6\end{array}$ & $\begin{array}{l}\text { Tarameters satura- } \\
\text { tion rule optimized } \\
\text { to best fit } 160 \\
\text { nonlinear GYRO } \\
\text { simulations }\end{array}$ \\
\hline$E \times B$ & $\begin{array}{l}\text { Self-consistently } \\
\text { through modified } \\
\text { eigenfunctions }\end{array}$ & $\begin{array}{l}\text { Included in spectral } \\
\text { shift of saturated } \\
\text { potential based on } \\
\text { GYRO nonlinear } \\
\text { results }\end{array}$ \\
\hline Fitted parameters & $\begin{array}{l}\text { One, such that, for } \\
\text { the GA standard } \\
\text { case, QuaLiKiz } \\
\text { ion energy flux } \\
\text { matches the non- } \\
\text { linear GYRO ion } \\
\text { energy flux }\end{array}$ & $\begin{array}{l}\text { All of the pa- } \\
\text { rameters were } \\
\text { determined by } \\
\text { fitting to linear and } \\
\text { non-linear theory }\end{array}$ \\
\hline Verification & $\begin{array}{l}\text { Against nonlinear } \\
\text { GYRO, GKW and } \\
\text { GENE simulations } \\
\text { see table III }\end{array}$ & $\begin{array}{l}\text { Against } 1799 \text { lin- } \\
\text { ear gyrokinetic } \\
\text { GKS runs and } 160 \\
\text { nonlinear GYRO } \\
\text { runs }\end{array}$ \\
\hline
\end{tabular}


TABLE II: Comparison of QuaLiKiz and TGLF frameworks.

\section{QUALIKIZ LINEAR GYROKINETIC DISPERSION RELATION}

In QuaLiKiz, the eigenvalues are solutions of a linear gyrokinetic equation and the eigenfunctions are solutions of the fluid limit of this equation. All unstable eigenvalues are found thanks to a Nyquist like method. Both the eigenvalues and the eigenfunctions have been benchmarked with more complete $\delta f$ gyrokinetic codes. In the review spirit of this short paper, the main approximations are presented and, for more exhaustive explanation, the adequate references are provided.

\section{A. The approximated QuaLiKiz linearized Vlasov equation}

In the core of tokamak plasmas, the measured density fluctuation level is well below $10 \%$ [41]. This validates assuming a background Maxwellian distribution function $f_{0}$ with a small perturbation $\delta f$. Thus, for each species $s, f_{s}=$ $f_{0, s}+\delta f_{s}$ with $f_{0, s}=n_{s} \frac{1}{\left(2 \pi m_{s} T_{s}\right)^{3 / 2}} e^{-E / T_{s}}$, with $T_{s}$ the temperature, $n_{s}$ the density and $m_{s}$ the mass of the considered species. $E$ is the kinetic energy of a particle at the thermodynamical equilibrium. When treating a rotating plasma in the laboratory frame, $E$ writes $E=\frac{m_{s}\left(v_{\|}-U_{\|}\right)^{2}}{2}+\frac{m_{s} v_{\perp}{ }^{2}}{2}$, with $U_{\|}$the bulk parallel rotation of the plasma. $v_{\|}$and $v_{\perp}$ are respectively the velocities parallel and perpendicular to the magnetic field $B$. In QuaLiKiz, the low Mach number limit is assumed.

The electrostatic limit is assumed in QuaLiKiz. This limit is valid for low $\beta$ plasmas. The total electrostatic potential is written as $\phi=\phi_{0}+\delta \phi$.

The Vlasov equation is linearized assuming harmonic perturbations such that $\delta f=\sum_{\vec{n} \omega} f_{\vec{n} \omega}(\vec{J}) e^{i(\dot{\vec{n} \vartheta}-\omega t)}$ and $\delta \phi=$ $\sum_{\vec{n} \omega} \phi_{\vec{n} \omega}(\vec{J}) e^{i(\vec{n} \dot{n} \vartheta-\omega t)}$ :

$$
f_{\vec{n} \omega}(\vec{J})=-\frac{f_{0}(\vec{J})}{T_{s}}\left(1-\frac{\omega-\vec{n} \cdot \vec{\omega}_{s}^{*}-\vec{n} \cdot \vec{\omega}_{E}}{\omega-\vec{n} \cdot \vec{\Omega}_{J}+i 0^{+}}\right) e_{s} \phi_{\vec{n} \omega}(\vec{J})
$$

$\vec{J}$ and $\vec{\vartheta}$ are respectively the action and angle variables $[42,43]$ and $\vec{n}$ is the associated wave vector. $\omega$ is the frequency of the perturbation $\delta f, e_{s}$ is the charge of the species $s$. The small positive imaginary part $i 0^{+}$is needed to insure the causality property such that the fluctuations cancel out for a time $t$ tending towards $-\infty \cdot \vec{n} \cdot \vec{\Omega}_{J}=\vec{n} \cdot \frac{d \vec{\vartheta}}{d t}$ is the drift frequency representing the forces felt in the tokamak electromagnetic field, it includes the $\nabla B$ and curvature drifts as well as the parallel dynamics, $k_{\|} v_{\|} \cdot \vec{n} \cdot \vec{\omega}_{E}=\vec{n} \cdot \frac{e_{s} d \phi}{T_{s} d \vec{J}}$ is the $E \times B$ drift and $\vec{\omega}^{*}$ is the diamagnetic frequency which represents the instability drive term since it expresses the departures from thermody- namical equilibrium of the quantities $T_{s}, n_{s}$ and $U_{\|}$such that:

$$
\begin{array}{r}
\vec{\omega}_{s}^{*}=T_{s} \frac{1}{n_{s}} \frac{d n_{s}}{d \vec{J}}+ \\
T_{s}\left(\mathcal{E}-\frac{3}{2}-\frac{U_{\|}}{v_{T_{s}}}\left(\frac{2 v_{\|}-U_{\|}}{v_{T_{s}}}\right)\right) \frac{1}{T_{s}} \frac{d T_{s}}{d \vec{J}}+ \\
2 T_{s}\left(\frac{v_{\|}-U_{\|}}{v_{T_{s}}}\right) \frac{d U_{\|}}{d \vec{J}} \frac{1}{v_{T_{s}}}
\end{array}
$$

with $\mathcal{E}=\frac{\frac{m_{s} v_{\|}^{2}}{2}+\frac{m_{s} v_{\perp}^{2}}{2}}{T_{s}}$ and $v_{T_{s}}$ such that $T_{s}=\frac{1}{2} m_{s} v_{T_{s}}^{2}$. In the electrostatic case, relevant wavelengths being larger than the Debye length, the dispersion relation is closed by the weak formulation of the quasineutrality condition :

$$
\sum_{s} \mathcal{L}_{s}(\omega, \vec{n})=0
$$

where $\mathcal{L}_{s}$ is the particle Lagrangian defined for each species s by $\mathcal{L}_{s}(\omega, \vec{n})=\int d^{3} \vec{x}\left(-\rho_{s}(\omega, \vec{n}) \phi^{*}(\omega, \vec{n})\right)$. This quasineutrality weak formulation is exact in the case of a unique eigenfunction [44].

Combining the weak variational formulation, equation (3), to the linearized Vlasov equation (1), one obtains the dispersion relation:

$$
\sum_{s} \frac{e_{s}^{2} f_{0}^{s}}{T_{s}}\left[\left\langle\phi_{\vec{n} \omega} \phi_{\vec{n} \omega}^{*}\right\rangle-\left\langle\frac{\omega-\vec{n} \cdot \vec{\omega}^{*}-\vec{n} \cdot \vec{\omega}_{E}}{\omega-\vec{n} \cdot \vec{\Omega}+i 0^{+}} \phi_{\vec{n} \omega} \phi_{\vec{n} \omega}^{*}\right\rangle\right]=0
$$

where $\langle\ldots\rangle=d^{3} \vec{\vartheta} d^{3} \vec{J} \ldots$, hence equation (4) leads to 6D integrals.

Here the abiabatic part is summed to the non-adiabatic one. An alternative formulation of the dispersion relation isolates the polarization term and the gyrocenter part [45].

The $6 \mathrm{D}$ dispersion relation can be reduced down to $5 \mathrm{D}$ by toroidal axisymmetry, and then down to $4 \mathrm{D}$ by the gyrokinetic approximation $\omega / \omega_{c} \ll 1$, where $\omega_{c}$ is the cyclotron frequency. This introduces a Bessel function in front of the nonadiabatic term and removes the gyroangle. Then, it reduces further down to 3 through the strong ballooning approximation. This approximation takes advantage of the fact that the dynamics along the field line is faster than the dynamics across the field line leading to $k_{\|} \ll k_{\perp}[43,46,47]$. The fluctuating electrostatic potential is hence rewritten as the product of slowly and rapidly varying functions: $e^{-i n(\varphi-q(x) \theta)} \phi_{n \omega}(\theta)$, where $\varphi$ is the toroidal angle, $\theta$ an extended poloidal angle and $q$ the safety factor. By utilizing the analytical Fried and Conte energy integrals the dispersion relation is further reduced to 2D. To simplify further, the trapped and passing particles are integrated separately using the circular concentric large aspect ratio magnetic equilibrium $s-\alpha$ [48, 49]. For passing particles, since $v_{\|} \gg v_{\perp}$, a pitch angle averaged transit frequency as well as curvature and $\nabla B$ drift frequencies are performed. For trapped particle, the bounce frequency being larger than $\omega$, a bounced average is performed. The trapped curvature and $\nabla B$ drift frequency is averaged over $\theta$. This approximation leads to an underestimation of Trapped Electron Modes in QuaLiKiz at intermediate wave numbers $\left(k_{\theta} \rho_{s} \simeq 0.5-10\right)$. 
A Krook type collision operator is taken into account $[50,51]$ for trapped electrons only. Collisions on trapped ions and passing particles are neglected. QuaLiKiz dispersion relation accounts for trapped and passing ions and electrons, i.e. it covers the ITG-TEM and ETG ranges. An arbitrary number of tracers or active ions can be accounted for [35]. The detailed dispersion relation can be found in [43].

\section{B. Validating the fluid eigenfunctions}

In the variational approach, an error $\epsilon$ in estimating the eigenfunction will lead to a smaller error, $\epsilon^{2}$, on the eigenvalue, allowing for a higher tolerance in approximations for the eigenfunction determination. The eigenfunction $\phi_{n \omega}(\theta)$ is hence derived separately in the fluid limit, which corresponds to: $\omega \gg \vec{n} \cdot \vec{\Omega}$. The fluid limit dispersion relation is developed analytically, details can be found in [17, 42, 43].

The validity of QuaLiKiz fluid eigenfunctions was successfully assessed by comparing to self-consistent gyrokinetic eigenfunctions. For consistency with QuaLiKiz, all direct comparisons are realized with the $s-\alpha$ equilibrium using $\alpha=0$ in either GKW, GYRO or GENE. Without rotation and $\mathbf{E} \times \mathbf{B}$, the QuaLiKiz Gaussian width agrees within a few $\%$ with the gyrokinetic GYRO width [50]. Nonetheless, wide oscillating eigenfunctions are reported in GENE/GKW for simultaneously low magnetic shear and wave number [52] and in large $k_{\theta}$ TEM dominated regimes [17]. The influence of $u_{\|}$ and $\nabla u_{\|}$on the eigenfunction shift is also successfully benchmarked against GKW in [17]. Moreover, the $\mathbf{E} \times \mathbf{B}$ shear leading to the expected asymmetric eigenfunctions is observed in QuaLiKiz [17].

\section{Searching for the eigenvalues}

QuaLiKiz is written in Fortran 90. Open source modules are used for the Fried and Conte integrals, the Bessel functions, the elliptical integrals and the adaptive 1D and 2D integration methods.

To find the eigenvalues of the QuaLiKiz dispersion relation, the Davies method is used [53]. Within a given contour in the complex plane, this method can determine the number of eigenvalues as well as an estimate of their values. Their precise value is then refined using a standard Newton's method. This numerical scheme was recently further optimized [35].

\section{Linear benchmark of the growth rates}

The first benchmark was presented in [42] and showed that the stabilizing impact of $Z_{\text {eff }}$ was found in both QuaLiKiz and GS2. Following the implementation of collisions on trapped electrons in QuaLiKiz [50], their stabilizing impact on TEM was tested against GS2 [54]. More recently, QuaLiKiz growth rates were compared to GENE over a magnetic shear (s) scan [52]. In QuaLiKiz the stabilization for negative values of $s$ is overestimated. It could be due to the pitch angle averaged transit frequency for passing particles leading to an underestimation of the slab branch. In the presence of finite rotation and/or $E \times B$ shear QuaLiKiz was extensively benchmarked against GKW [17].

In most cases the order of magnitude of the growth rate is correct within a few tens of $\%$. Overall, QuaLiKiz tends to underestimate the growth rates due to narrower eigenfunctions in $\theta$.

\section{THE QUASILINEAR FORMULATION AND ITS VALIDITY}

In this section, the quasilinear flux formulation is briefly presented. Then the validity of the quasilinear approximation is extensively discussed. The QuaLiKiz formulation of the saturated potential is deduced from the quasilinear validity exploration. Note that, as previously, a brief and wide overview is proposed here. The reader interested in further details is guided towards additional references.

\section{A. The quasilinear flux formulation}

The quasilinear approach can be used if a time scale, $\tau$, exists such that $1 / \gamma<\tau<T_{0}$, where $T_{0}$ is the equilibrium time scale. The quasilinear flux, e.g. for particle flux, is defined as $\Gamma_{s}=\left\langle\delta n_{s} \delta V_{E \times B}\right\rangle$, where $\langle\ldots\rangle$ is a flux surface average and time average over $\tau$. For electrostatic turbulence using the linearized Vlasov equation (1), the particle flux can be expressed as the product of a linear plasma response with a saturated electrostatic potential as follows:

$$
\begin{array}{r}
\Gamma_{s}=\operatorname{Re}\left\langle\delta n_{s} \frac{i k_{\theta} \delta \phi}{B}\right\rangle \\
=\sum_{\vec{n}, \omega} n \operatorname{Im}\left\langle\vec{n} \cdot \frac{d f_{0, s}(\vec{J})}{d \vec{J}} \frac{1}{\omega-\vec{n} \cdot \overrightarrow{\Omega_{J}}+i 0^{+}} e_{s}^{2}\left|\phi_{\vec{n} \omega}(\vec{J})\right|^{2}\right\rangle \\
\propto \sum_{\vec{n}, \omega}\left\langle R_{\text {lin }}(\omega, \vec{n})\left|\phi_{\vec{n} \omega}\right|^{2}\right\rangle
\end{array}
$$

Note that energy, and momentum transport fluxes are similarly derived [17, 43]. Here the time averaging was carried out and the frequency $\omega$ is taken as the real part of the solution of the dispersion relation equation (4). Nonlinear effects tend to broaden the frequency spectrum, hence a finite value will be used instead of $0^{+}$, as discussed below. Equation (5) is valid if the two following linked assumptions hold: i) the linear properties are maintained in the saturated phase and ii) the random-walk assumption holds. In the following, both assumptions are shown to be valid over a large range of parameters. Nonetheless, the amplitude and spectral shape of the saturated potential in equation (5) is not set by linear physics. This needs to be tuned by nonlinear simulations, turbulence measurements, and physical intuition. This is a key attribute of all quasilinear transport models. 


\section{B. Testing the quasilinear approximation validity and building} the saturated potential

\section{The Kubo number}

Historically, the standard quasilinear theory was elaborated for test particles [55]. A condition of validity for this quasilinear framework is that the particles should not be trapped in the field. This means that decorrelation time of the potential should be shorter than the eddy turn-over time. The ratio of these two times is known as the Kubo number [56, 57]. Using gyrokinetic nonlinear codes, these times were derived and compared to each other for ETG turbulence [58] and for ITG-TEM turbulence [52, 59]. For all cases, no field trapping is reported, hence a Kubo number below 1. Therefore, it is demonstrated that well developed turbulence for typical tokamak plasma parameters is such that random walk processes do take place and can be modeled by the quasilinear theory. This is an important feature of tokamak plasma turbulence. It is coherent with results reported in the introduction regarding the overall success of quasilinear models in reproducing temperature profiles within around $15 \%$ averaged RMS error [29].

\section{The frequency spectrum}

As mentioned in equation (5), a finite positive imaginary part at the denominator guarantees causality. In the case of $0^{+} \rightarrow 0$, the term $\operatorname{Im}\left(\frac{1}{\omega-\vec{n} \cdot \Omega_{J}+i 0^{+}}\right)$tends towards a Dirac function $-\pi \delta\left(\omega-\vec{n} \cdot \overrightarrow{\Omega_{J}}\right)$. To avoid these singularities and obtain continuous frequency spectra, a finite positive imaginary part is required. This is often referred as the renormalized quasilinear theory, also called the Resonance Broadening Theory [60]. Indeed, if a finite imaginary part $\nu_{Q L}$ is assumed instead of $0^{+}$, one obtains:

$$
\operatorname{Im} \frac{1}{\omega-\vec{n} \cdot \overrightarrow{\Omega_{J}}+i \nu_{Q L}}=\frac{\nu_{Q L}}{\left(\omega-\vec{n} \cdot \overrightarrow{\Omega_{J}}\right)^{2}+\nu_{Q L}^{2}}
$$

which is a Lorentzian of width $\nu_{Q L}$. Two questions have now to be answered: is the Lorentzian an appropriate shape, and what is the value of its width, $\nu_{Q L}$.

In tokamak plasmas, the frequency spectrum is measured by Doppler reflectometry and its shape can be compared to Lorentzian as well as Gaussian fits. The same procedure can carried out for a GYRO nonlinear frequency spectrum [61]. The density fluctuations are computed at the outboard mid-plane, according to the disgnostic line of sightNeither of the two shapes are correct, as illustrated by figure 1. The turbulence cannot be approximated by either a purely diffusive (Lorentzian) or convective (Gaussian) model. Nevertheless, both fits capture the majority of the spectrum (note that the figure is in a semi-log scale), and although not exact, a Lorentzian fit, as chosen in QuaLiKiz is acceptable.

The width $\nu_{Q L}$ can be estimated from a crude approximation assuming that for each mode, the linear growth rate competes
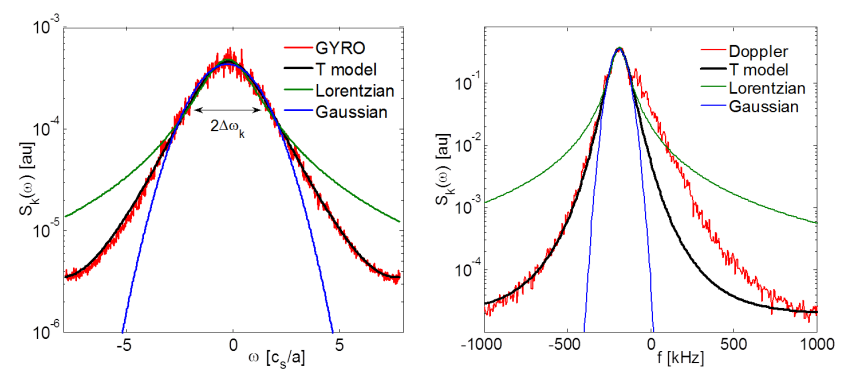

FIG. 1: At $\rho=0.7$, TS39596, experimental parameters given in [62]. Density fluctuation spectra at $k_{\theta} \rho_{s}=0.82$ in red, compared to a Lorentzian fit in green, a Gaussian fit in blue and a model test $\mathrm{T}$ function [57] in black. Left hand side : frequency spectrum from GYRO nonlinear simulation. Right hand side: experimental Doppler reflectometry spectrum. From [61].

with the nonlinear damping. Within this picture, the nonlinear decorrelation takes place at the same rate that the free energy is replenished [63], leading to:

$$
\nu_{Q L}=\gamma
$$

This hypothesis was tested in nonlinear simulations [52, 61]. Figure 2 shows that for $k_{y} \simeq k_{\theta} \rho_{s}<0.2$, the linear growth rate of the most unstable mode represents rather well the nonlinear width. Since most of the transport occurs at low $k_{\theta}$, the QuaLiKiz choice if a Lorentzian of width $\gamma$ is a reasonable assumption for most cases. Indeed, on the semilog plots of figure 1 more than $80 \%$ of the spectrum is captured by either a Lorentzian or a Gaussian shape. However, an increased frequency broadening at low magnetic shear was reported as being likely the result of non-linear decorrelation mechanisms, such as zonal flows [52]. This was dealt by including an additional shear dependent normalization factor to QuaLiKiz fluxes, as discussed in the following section, see equation (9). The frequency around which the nonlinear spectrum is centered was also compared to the linear eigenmode frequency $\omega$. As illustrated by figure 3 , a very good agreement is observed at scales dominating the transport: $k_{\theta} \rho_{s} \leq 0.4$ [61]. Nonetheless, recently quasi-coherent modes were observed in reflectometry spectra [64] and successfully identified as a TEM signature in GENE nonlinear simulation [65]. Such modified frequency spectra should be accounted for in a revisited renormalized quasilinear fluxes.

\section{Testing the validity of the linear response}

By comparing nonlinear and linear fluxes in different ways, the validity of the linear Vlasov equation for $R_{\text {lin }}$ in $\Gamma_{s} \propto$ $\sum_{\vec{n}, \omega}\left\langle R_{\text {lin }}(\omega, \vec{n})\left|\phi_{\vec{n} \omega}\right|^{2}\right\rangle$ can be investigated. The two approaches are 1/ comparing the nonlinear and quasilinear phases between the transported quantities and the fluctuating potential; 2/ comparing the fluxes either quasilinear or nonlinear divided by the same saturated potential, typically the nonlinear saturated potential. Both are reviewed here. A phase between a transported quantity and the fluctuating 


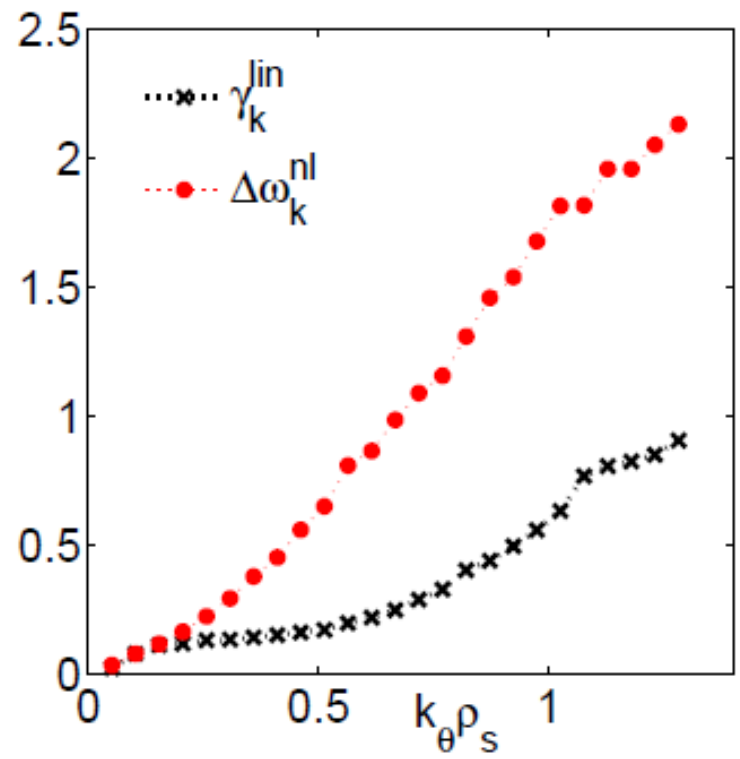

FIG. 2: $k_{\theta}$ spectrum of the linear growth rates and of the nonlinear frequency widths. The results refer to nonlinear and linear (most unstable mode) simulations using GYRO on TS39596 at $r / a=0.7$ [62].

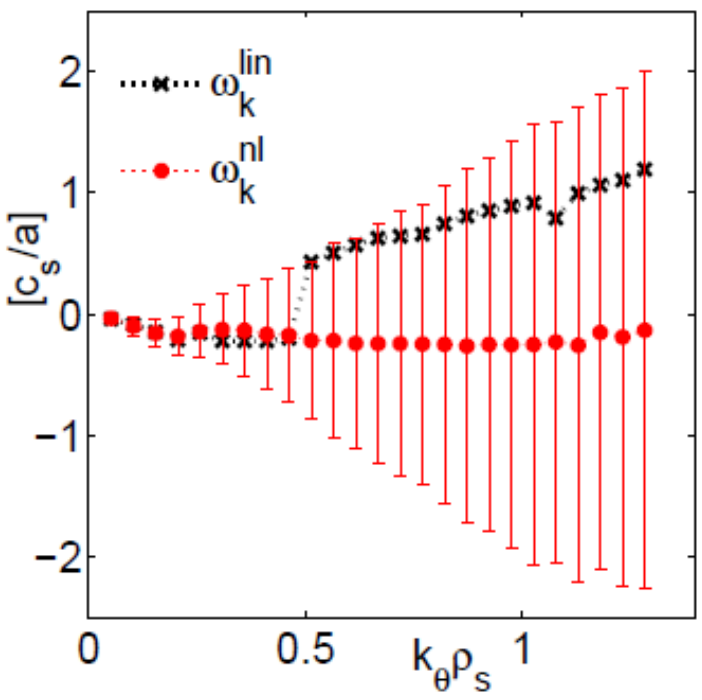

FIG. 3: $k_{\theta}$ spectrum of the frequencies of the linear most unstable mode and of the nonlinear frequencies (the bars indicate the statistical variance from the mean value) [61]. The results refer to nonlinear and linear (most unstable mode) simulations using GYRO on TS39596 at $r / a=0.7$ [62].

potential, for example between $\delta n_{s}$ and $\delta \phi$ for particles, different from 0 or $\pi$ is responsible for a finite level of transport. The nonlinear phases can be extracted from nonlinear simulations $[59,66]$. The linear phase corresponds to the phase of the complex expression of $R_{\text {lin }}(\omega, k)$. A good agreement has been reported for $k_{\theta} \rho_{s} \leq 0.4$ in the case of pure TEM tur- bulence [66] and for coupled ITG-TEM turbulence [59, 67]. It demonstrates that for low $k_{\theta}$ the linear response captures properly the phase between transported quantities and fluctuating potential.

In a nonlinear simulation, over a significant time window of the saturated phase, for each wave number $k$, the flux surface average nonlinear flux $\Gamma_{k}^{N L}$ normalized to the saturated potential $\left|\delta \phi_{k}\right|_{N L}^{2}$ on a flux surface can be defined as a nonlinear transport weight $w_{k}^{N L}$ such that:

$$
w_{k}^{N L}=\frac{\Gamma_{k}^{N L}}{\left|\delta \phi_{k}\right|_{N L}^{2}}
$$

$w_{k}^{N L}$ amplitude can be compared to the quasilinear weight, $w_{k}^{Q L}$, which is proportional the linearized Vlasov equation, $R_{\text {lin }}$, as expressed in equation (5). The weight amplitudes are compared using the nonlinear saturated potential for both the nonlinear and quasilinear weights such that : $w_{k}^{Q L}=\frac{\Gamma_{k}^{Q L}}{\left|\delta \phi_{k}\right|_{N L}^{2}}$ [67]. It is found that the ratio $\frac{w_{k}^{Q L}}{w_{k}^{N L}}$ is around 1.4 in various cases: the ITG dominated GA standard case as well as two TEM dominated regimes with GYRO [67], with adiabatic electrons for a Tore Supra $\mathrm{L}$ mode case at $r / a=0.4$ using both GYSELA and GYRO [59]. This value is independent of $k_{\theta} \rho_{s}$ in the low $k$ range dominating the transport. This comparison allows to further validates the linear response $R_{\text {lin }}$. Nonetheless, as $s$ is reduced from 1 down to 0.1 , the transport weight overage is increased from 1.4 up to larger values, even at low $k_{\theta}$ [52]. Another departure from 1.4 is observed in the case of strong inward particle fluxes with GYRO [67] and GKW [39] where the inward particle flux is stronger nonlinearly than quasilinearly. The origin of this 1.4 factor could be linked to nonlinear transfer of energy through zonal flows or through transfer towards stable eigenmodes [68]. To strengthen these hypotheses, a more theoretically robust renormalized quasilinear theory is required as the behavior at low $s$ and strong inward particle fluxes reported above further illustrates. In the absence of more rigorous theory, an ad-hoc additional $s$ dependent normalization factor was included on top of the 1.4 factor for $|s|<0.6$, in the form of:

$$
2.5(1-|s|)
$$

Despite the lack of understanding of the theoretical origin of the 1.4 factor adjusted for $|s|<0.6$, it has allowed QuaLiKiz to robustly reproduce GENE, GYRO or GKW nonlinear fluxes over a wide range of parameters as summarized in table III, including the vicinity of the temperature gradient threshold. The only remaining identified disagreement between quasilinear and nonlinear fluxes occurs for the particle fluxes in cases of hollow density profiles [39]. See also the next section for further discussion of these comparisons.

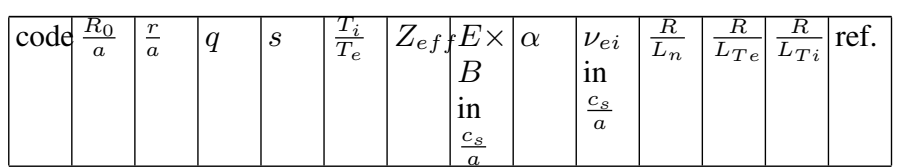




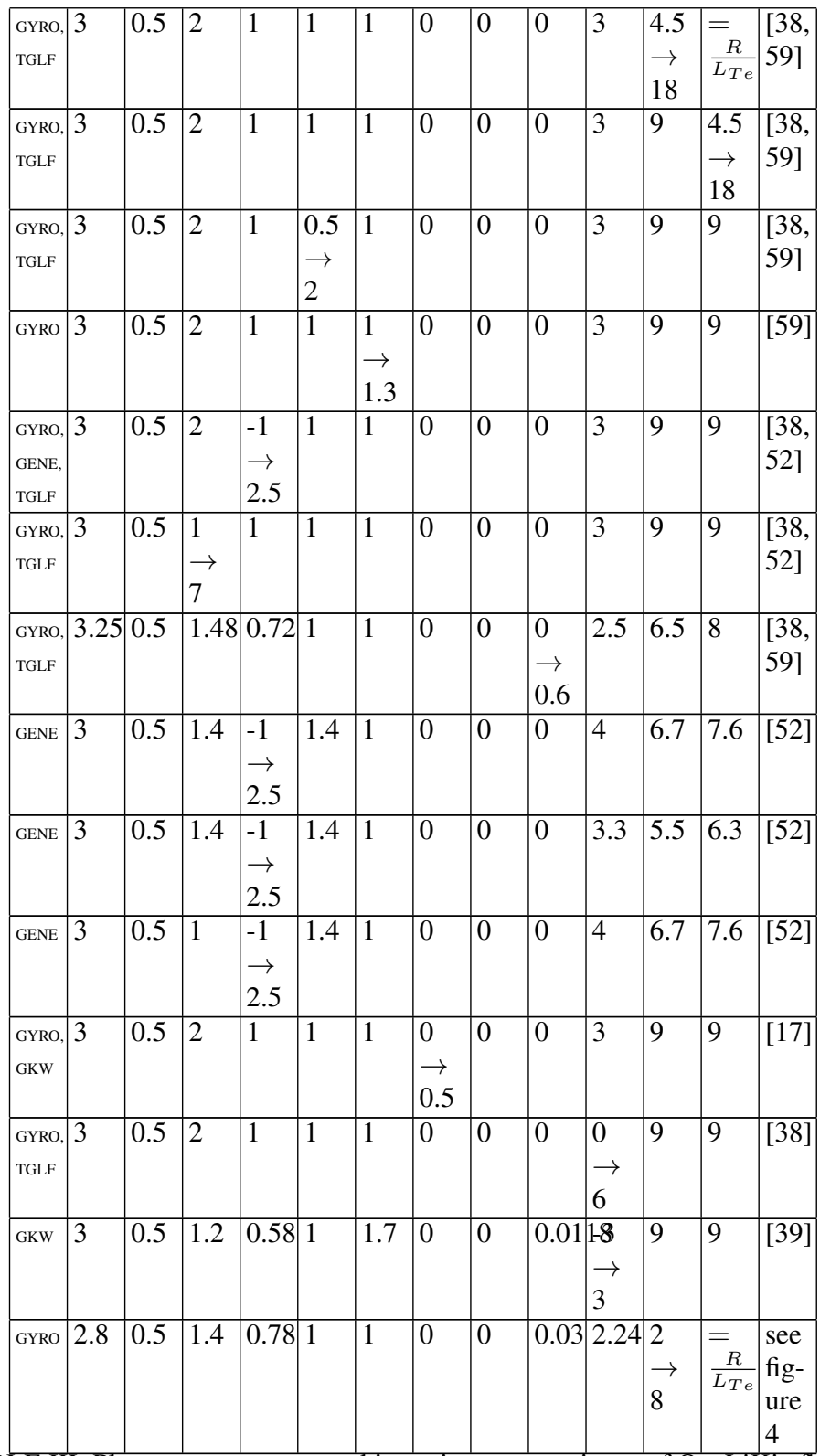

TABLE III: Plasma parameters used in various comparisons of QuaLiKiz fl against nonlinear gyrokinetic codes (GYRO, GKW and GENE) and TGLF.

4. The saturated potential level and k spectrum in QuaLiKiz

On most experiments [69-71], the density fluctuation wavenumber spectrum : $S\left(k_{\perp}\right)=\left|\frac{\delta n\left(k_{\perp}\right)}{n}\right|^{2}$ shows a decay for $k_{\perp} \rho_{s} \geq 0.2$ such that: $S\left(k_{\perp}\right) \simeq k_{\perp}^{\alpha_{\perp}}$ with $\alpha_{\perp}=-3.5 \pm 0.5$. This was also observed on a Tore Supra ohmic L mode pulse using two reflectometers which agreed well with nonlinear GYSELA and GYRO simulations confirming isotropic $k$ spectra with a decay of $\alpha_{\perp} \simeq-3$ [62]. Moreover this decay is successfully recovered by the shell model [72]. This choice is hence retained in QuaLiKiz.
Now that the slope above the maximum potential is determined, it is necessary to set the $k$ value of the maximum potential: $k_{\max }$. It is chosen such that the effective diffusivity, $D_{\text {eff }}$, follows the mixing length rule:

$\max \left(D_{e f f} \simeq \frac{R \Gamma_{s}}{n_{s}}\right)_{k_{\max }}=\frac{R}{n_{s}} \frac{k_{\theta}}{B} \frac{n_{s} e_{s}}{T_{s}}\left|\tilde{\phi}_{n \omega}\right|_{k_{\max }}^{2}=\max \left(\frac{\gamma}{\left\langle k_{\perp}^{2}\right\rangle}\right.$

$k_{\text {max }}$ corresponds to the $k$ value at which $\frac{\gamma}{\left\langle k_{\perp}^{2}\right\rangle}$ is maximum as proposed in [66].

Here $-\nabla n_{s} \simeq n_{s} / R$. By making use of the mixing length rule, a random Gaussian statistics for the saturated electrostatic potential is assumed [57]. This result is based on the picture that the nonlinear damping rate, $D_{\text {eff }}\left\langle k_{\perp}^{2}\right\rangle_{k_{\max }}$ balances the linear growth rate $\gamma$ [73]. Hence $D_{\text {eff }}\left\langle k_{\perp}^{2}\right\rangle_{k_{m}}$ is equal to the previously introduced $\nu_{Q L}$ and is modified in presence of zonal flows for example [74].

The choice for $\left\langle k_{\perp}^{2}\right\rangle$ is based on both experimental observations and nonlinear simulation results. It should lead to a maximum $\left|\phi_{n \omega}\right|^{2}$ around $k_{\theta} \rho_{s} \simeq 0.2$ as reported by numerous nonlinear simulations [62], and as observed with Beam Emission Spectroscopy [75]. It should also depend on $q$ as observed in nonlinear simulations $[66,76]$. A pertinent choice for $\left\langle k_{\perp}^{2}\right\rangle$ combining these two aspects was proposed by $[7,66,77]$. It is here extended to account for the MHD $\alpha$ parameter on the curvature drift as well as the magnetic shear $s$ [8] such that:

$$
\left\langle k_{\perp}^{2}\right\rangle=k_{\theta}^{2}\left[1+(s-\alpha)^{2}<\theta^{2}>\right]
$$

with:

$$
<\theta^{2}>=\frac{\int \theta^{2}\left|\phi_{n \omega}(\theta)\right|^{2} d \theta}{\int\left|\phi_{n \omega}(\theta)\right|^{2} d \theta}
$$

The eigenfunction $\left|\phi_{n \omega}(\theta)\right|^{2}$ is a shifted Gaussian, accounting for finite $\mathbf{E} \times \mathbf{B}$ and rotation. Hence, symmetry breaking in $\left\langle k_{\perp}^{2}\right\rangle$ is enacted through the imaginary part of the eigenfunction shift and the real part of the mode width, the latter being proportional to the growth rate found in the fluid model.

The expression for $\left\langle k_{\perp}^{2}\right\rangle$, proposed in [8], was revisited in [52] to improve QuaLiKiz fluxes estimation at low magnetic shear. It reads:

$\left\langle k_{\perp}^{2}\right\rangle=k_{\theta}^{2}+k_{r}^{2}$

$k_{r}=$

$\sqrt{k_{\theta}^{2} \hat{s}^{2}\left\langle\theta^{2}\right\rangle}+\frac{0.4 \exp (-2 \hat{s})}{\sqrt{q}}+1.5\left(k_{\theta}-0.2 / \rho_{s}\right) H\left(k_{\theta}-0.2 / \rho_{s}\right)$

At low magnetic shear, the $k_{r}^{2}=k_{\theta}^{2} \hat{s}^{2}\left\langle\theta^{2}\right\rangle$ resulting from magnetic field line shearing, is underestimated with respect to nonlinear $k_{r}$ [52]. The factor $\frac{0.4 \exp (-2 \hat{s})}{\sqrt{q}}$ was found to represent well the non linear isotropization at low $s$. Finally, the term $1.5\left(k_{\theta}-0.2 / \rho_{s}\right) H\left(k_{\theta}-0.2 / \rho_{s}\right)^{2}$ ( $H$ is the Heaviside function) is present to correctly capture the approximate isotropy at high wave-numbers, but typically plays a negligible role in setting the maximum value of $\gamma /\left\langle k_{\perp}^{2}\right\rangle$. 
To conclude, it should be stressed that the amplitude of the saturated potential peak is normalized in QuaLiKiz by a unique constant, such that the quasilinear ion energy flux for the GAstandard case reproduces the nonlinear result. This QuaLiKiz saturated potential level and $\mathrm{k}$ spectrum were successfully compared to GKW for three values of the $\mathbf{E} \times \mathbf{B}$ shear [17]. Despite some quantitative differences, the nonlinear flux quenching with $\mathbf{E} \times \mathbf{B}$ shear is captured qualitatively with a shifted eigenfunction calculated in the linear fluid limit. Moreover the linear $k_{\theta}$ dependence up to the maximum, the value of $k_{\max }$ and the slope in $k_{\theta}^{-3}$ above $k_{\max }$ all reproduce fairly well the nonlinearly saturated potential $\mathrm{k}$ spectra.

\section{COMPARING QUALIKIZ QUASILINEAR FLUXES}

To construct QuaLiKiz fluxes, two elements are required: the linear response $R_{\text {lin }}$ and the saturated electrostatic potential $\left|\phi_{k \omega}\right|^{2}$. $R_{l i n}$ is the linear gyrokinetic dispersion relation developed in the framework discussed in section 2 and summarized in table II. The saturated potential has its maximum value constrained by the mixing length rule see equation (10), and its $\mathrm{k}$ spectrum decreases as $k^{-3}$ as reviewed in section 3. The Lorentzian frequency spectrum, equation (6), is obtained by replacing $0^{+}$in equation (5) by the linear growth rate $\gamma$. The sum over $k$ is typically done over 20 values from $k_{\theta} \rho_{s}=0.05$ up to 2 . In QuaLiKiz, all eigenvalues contributions to the flux are summed. Finally, the saturated potential level is adjusted by equaling QuaLiKiz ion energy flux with GYRO nonlinear ion energy flux for the GA standard case. This only adjustment is done once and for all. The fact that QuaLiKiz fluxes reproduces well nonlinear fluxes for the ion energy fluxes using other sets of parameters, as well as electron energy fluxes, particle fluxes and angular momentum fluxes proves the validity of QuaLiKiz derivation as detailed further in this section. For a complete derivation of the quasilinear fluxes see [8] for particle, [17] for angular momentum and [43] for on overview.

\section{A. QuaLiKiz fluxes compared to nonlinearly computed fluxes}

QuaLiKiz ion and electron energy and particle fluxes without rotation nor $\mathbf{E} \times \mathbf{B}$ shear were extensively compared to nonlinear simulations as summarized in table III. Over a wide range of normalized temperature gradients: $4.5<R / L_{T i}=R / L_{T e}<13.5$ QuaLiKiz and GYRO agree within $15 \%[59,61]$. Showing that both the ratio between the transport channels and the parametric dependence are well captured by the quasilinear approach.

The collisionality impact on transport is a crucial test for quasilinear models. Indeed two effects are potentially at play: a collisional damping of zonal flows [78] not included in QuaLiKiz and a linear collisional TEM damping. Over an experimentally relevant range of collisionality [59], the linear TEM damping is found to be dominant and QuaLiKiz fluxes agree well with GYRO. In particular, the particle flux reverses direction as $\nu_{*}$ increases as expected [7].
A transition from TEM dominated to ITG dominated domains is realized by keeping fixed $R / L_{T e}=9.0$ and varying only the ion gradient $R / L_{T i}$ from 1 to 18 [59]. The electron energy fluxes are well matched; discrepancies are instead observed on the particle fluxes for strong ITG turbulence and for the ion energy flux both in the deep TEM and deep ITG regimes. Above $R / L_{T i}=13$, the QuaLiKiz overestimation can be ascribed to a more pronounced effect of zonal flows in the nonlinear saturation.

When approaching the critical temperature threshold, the impact of zonal flows in pure ITG turbulence was shown to lead to an upshift of the threshold referred to as the "Dimits upshift" [79]. It was, for the Cyclone base case and with adiabatic electrons, a shift in normalized temperature gradient units such that: $\Delta\left(R / L_{T}\right) \simeq 2$. In presence of kinetic electrons, it was reduced to $\Delta\left(R / L_{T}\right) \simeq 0.8$ [80]. To explore this region, a $R / L_{T}=R / L_{T e}=R / L_{T i}$ scan down to the linear threshold is performed using QuaLiKiz and GYRO as illustrated by figure 4 . The electrons are kinetic and the collisionality is finite as detailed in table IV. Close to the stability threshold the nonlinear runs are more demanding in term of resolution and their convergence should be carefully checked. Therefore, from figure 4, only an upper limit to the Dimits shift can be set such that $\Delta\left(R / L_{T}\right) \leq 0.7$. The reduced Dimits shift observed with kinetic electrons and finite collisionality is hence coherent with the overall success of quasilinear models in reproducing profiles within around $15 \%$ averaged RMS error $[29,38]$.

\begin{tabular}{|c|c|c|c|c|c|c|c|c|c|}
\hline$R_{0} / a$ & $r / a$ & $R / L_{n}$ & $q$ & $s$ & $T_{i} / T_{e}$ & $Z_{\text {eff }}$ & $\rho_{*}$ & $\beta$ & $\nu_{e i}$ in units of $c_{s} / a$ \\
\hline 2.8 & 0.5 & 2.24 & 1.4 & 0.78 & 1.0 & 1.0 & 0.006 & 0 & 0.03 \\
\hline
\end{tabular}

TABLE IV: Plasma parameters of $R / L_{T}=R / L_{T e}=R / L_{T i}$ scan of figure 4 .

In [52], the impact of $q$ and more specifically of magnetic shear $s$ was extensively investigated. The RMS error on the particle and energy fluxes between QuaLiKiz and the nonlinear GENE and GYRO simulations are for the ion energy flux $\sigma=0.26$, for electron energy flux $\sigma=0.33$ and particle flux $\sigma=1$. Where $\sigma$ is the root of the ratio between the sum of the squares of the differences between the quasilinear and nonlinear fluxes divided by the sum of the squares of the nonlinear fluxes.

Quasilinear angular momentum fluxes were also validated [17]. For an $\mathbf{E} \times \mathbf{B}$ scan at zero $U_{\|}$, the angular momentum flux corresponds to the residual stress. In absolute value, the momentum flux increases at first with $\gamma_{E}$ due the $\mathbf{E} \times \mathbf{B}$ shear asymmetrization of the eigenfunction. Then, as $\gamma_{E}$ increases further, the momentum flux is slowly reduced due to the turbulence quenching by the $\mathbf{E} \times \mathbf{B}$ shear. This qualitative trend is found for GYRO, GKW and QuaLiKiz [17]. Quantitatively, QuaLiKiz overestimates the momentum flux found with GKW by $\sim 50 \%$. The discrepancy between QuaLiKiz and GKW is related to the overestimation of the saturated potential amplitude at lower $k_{\theta} \rho_{s}$ and intermediate values of $\gamma_{E}$ in QuaLiKiz 


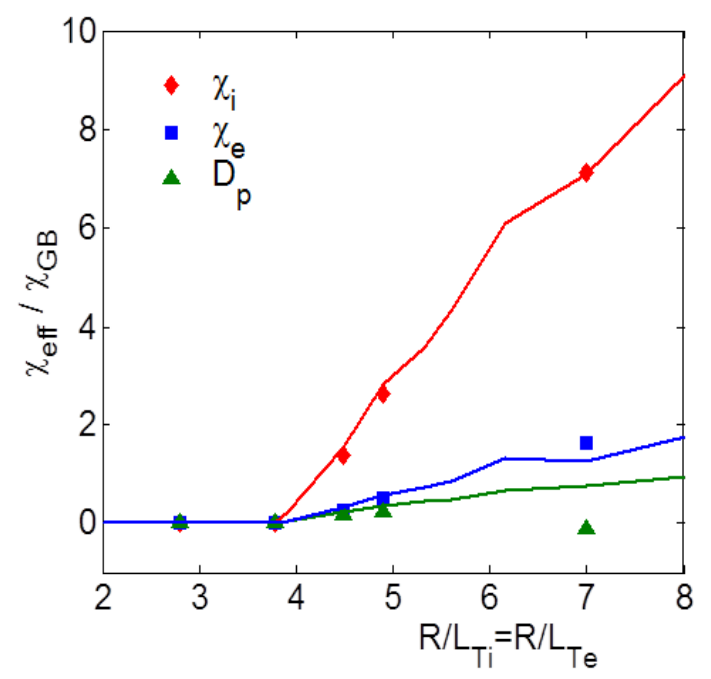

FIG. 4: Ion energy, electron energy and particle effective diffusivities from GYRO (points) and QuaLiKiz (lines) for the parameters of table IV plotted versus $R / L_{T}=R / L_{T e}=R / L_{T i}$.

as presented in the previous section. In QuaLiKiz the $\mathbf{E} \times \mathbf{B}$ shear is estimated assuming that the $\nabla_{r}\left(\nabla_{r}\left(P_{s}\right)\right)$ terms are negligible, hence assuming that $\nabla_{r} U_{\|}$is the dominant contribution. This approximation is not always justified, as in [81], nonlinear GYRO simulations show that the neglected terms can provide a residual stress comparable to the profile velocity shear contribution. Depending on the respective signs, the two contributions can be additive or oppose each other [52].

The effect of $\nabla u_{\|}$and $u_{\|}$on the momentum flux are analyzed with the aid of dimensionless quantities: the Prandtl number $\frac{\chi_{\|}}{\chi_{i}}$ and the pinch number $\frac{R V_{\|}}{\chi_{\|}}$. A normalized density gradient $R / L_{n}$ scan is chosen to illustrate this comparison [82, 83]. In [17], the QuaLiKiz Prandtl number is found to be close to 0.7 agreeing with quasilinear [84] and nonlinear GKWsimulations [83]. The QuaLiKiz pinch number $\frac{R V_{\|}}{\chi_{\|}}$is found to vary from -2 to -5 , with a strong correlation with $R / L_{n}$, as in [83]. This illustrates that the eigenfunction shift is a key element and that QuaLiKiz models correctly its impact on the momentum flux convective and diffusive parts.

\section{B. quasilinear fluxes vs experiments}

\section{Electron particle flux}

Following the overall success of quasilinear models in reproducing numerous features of the electron particle transport at a given time slice $[8,18,85,86]$, it is natural to now use such models in an integrated modeling framework to model density profile evolution. The experimental case on which the focus is made concerns high plasma current JET H-mode discharges $\left(I_{p}>2.0-2.5 \mathrm{MA}\right.$, depending on plasma shape), with naturally higher plasma densities for which NBI penetration is poorer [87]. It leads to hollow density profiles persist-
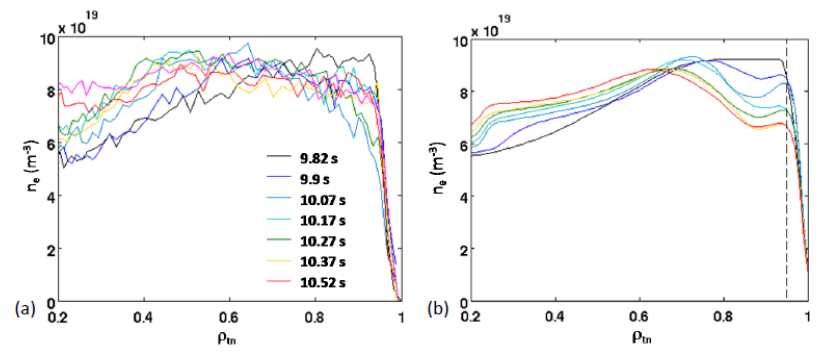

FIG. 5: Evolution of the density profile (a) from HRTS in the experimental JET pulse 79676 and (b) as obtained by the QuaLiKiz simulation in CRONOS. Different colors correspond to the times of the legend of fig. (a). The experimental density profile at $10.6 \mathrm{~s}$, after the first sawtooth, is included in the graph (a) in purple. The dashed line of (b) corresponds to the radial location of the imposed boundary conditions. From [39].

ing for timescales of several energy confinement times. When extrapolated towards ITER in D-T operation, such transient hollow density profiles lower the $\mathrm{L}$ to $\mathrm{H}$ power threshold and hence facilitates an early $\alpha$ central heating, necessary to maintain the $\mathrm{H}$ mode. To improve the accuracy of ITER extrapolation, the modeling of JET pulse by applying QuaLiKiz embedded in CRONOS was carried out [39]. Provided that the density pedestal is recovered, QuaLiKiz predicted hollow density profiles consistent with the experiment as illustrated by figure 5. The hollow profile is maintained since the diffusion - initially directed outward - is not counterbalanced by sufficient inward convection. Despite this success, more robust quasilinear models are required for hollow density profiles as mentioned earlier in this section, since in such cases the quasilinear fluxes underestimate the nonlinear inward fluxes. Also it is interesting to note that in an attempt to let evolve multiple transport channels simultaneously, a CRONOS simulation where the ion and electron energy fluxes were evolving together with the particles has also be performed. In this case, the hollow profile is filled faster in this case likely due to the fact that the temperatures are overestimated [39]. When investigating multiple transport channels at a time, the nonlinear interplay between them, with the sources and with the boundary conditions are extremely rich and complex. Thanks to faster first principle based tools such as TGLF and QuaLiKiz, this interplay should become a strong axis of research in the future.

\section{Impurity particle transport}

Impurity particle transport can be explored experimentally thanks to perturbation techniques [12, 88-90]. The impact of the impurity charge $Z$ was studied $[8,11,91,92]$. In Tore Supra, no dependence in $Z$ was found on the diffusivities [89], in agreement with QuaLiKiz. Concerning the inward convection, experimentally no $Z$ dependence was reported whereas it was expected from the modeling.

For impurity transport dominated by TEM turbulent transport, an influence of $R / L_{T e}$ is expected. This was studied in [12]. 
Within the uncertainties, QuaLiKiz reproduces the diffusion and convection of Nickel reasonably well as well as the increase of the diffusion with larger $R / L_{T e}$.

In ASDEX Upgrade, in the presence of central electron heating, average $Z$ impurities had either a weak or even a positive convection. This leads to weaker core impurity accumulation. The theoretical understanding of this mechanism is thought to be due to the passing electron compressibility term, leading to an outward convection in case of dominant TEM as reported in $[9,93]$. It is shown that an outward convection is obtained only if both $R / L_{T i}$ is low and $R / L_{T e}$ is high. To reproduce ASDEX Upgrade's result $R / L_{T e}$ has to be increased above its experimental value. This mechanism cannot explain results obtained in JET on Nickel transport [10]. It is important to note that such cases, deeply in the TEM regime, are accompanied by eigenfunctions extended along the field line (i.e. non-zero for $\theta= \pm \pi$, see appendix of [17]). In QuaLiKiz, the lowest order ballooning approximation, assuming null eigenfunctions at $\theta= \pm \pi$, is not adapted for such cases where complementary gyrokinetic simulations have to be carried out. It is also to note that, in Tore Supra dominantly electron heated pulses, such deep TEM regimes were not found and the experimentally measured impurity convection was never found directed outward.

\section{Energy flux}

For a wide range of parameter space the QuaLiKiz electrostatic assumption for ion energy flux is appropriate, including in L-modes as illustrated by figure 6 and in baseline H-modes [38] where the profiles predicted by QuaLiKiz and TGLF are shown to be similar. In the L mode ohmic Tore Supra case, figure 6, the temperature profiles are predicted by QuaLiKiz in CRONOS from the core to a normalized radius of 0.8 . In other cases, with high $\beta$ and/or significant fast ion pressure, such as in the hybrid $\mathrm{H}$ modes, the plasma conditions are close to the Kinetic Ballooning Modes limit. Under such condition, the nonlinear electromagnetic stabilization will be important [17] and QuaLiKiz will overpredict the fluxes [38]. This can be accounted for by changing the saturated potential rather than the linear part, since it is mostly a nonlinear effect.

The $\mathrm{L}$ mode edge was also reported as a region where both nonlinear and quasilinear gyrokinetic models failed in reproducing systematically measured turbulent transport levels [94-96]. Resistive Ballooning Modes were proposed as potential key players [27, 97]. RBM are not accounted for in QuaLiKiz. More recently the role of the bulk rotation direction was pointed out [98]. This effect will be investigated in future work using QuaLiKiz.

Towards the magnetic axis, experimentally an electron heat diffusivity larger than the neoclassical predictions is reported whereas gyrokinetic codes are often below the linear stability threshold. For example, on figure 6 , one can note that the modeled electron temperature values are above the measured ones for $r / a \leq 0.2$. To reconcile the modeling with the experimental observations, nonlinear turbulence spreading [99] should be investigated. ETG might also be a candidate, since
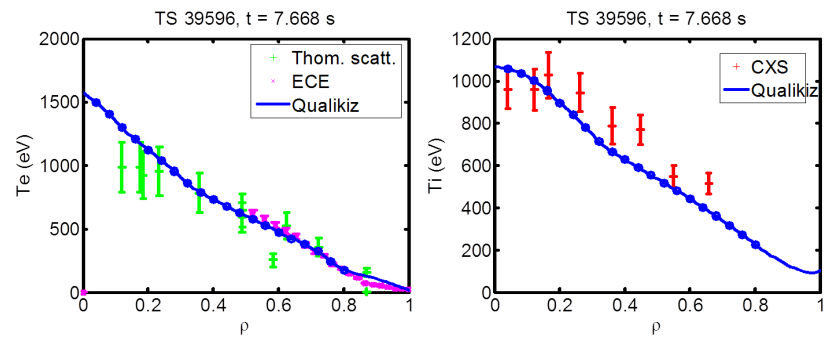

FIG. 6: Left hand side: Electron temperature profile measured by ECE and Thomson Scattering and QuaLiKiz prediction. Right hand side: Charge Exchange measured ion temperature and QuaLiKiz prediction. Tore Supra pulse 39596 [62].

recent multiscale simulations show that high $k$ ETG can nonlinearly be responsible for large increase of high $k$ turbulent fluxes [100].

\section{Angular momentum transport}

To determine experimentally the momentum convective term, modulations of the momentum sources are necessary. Such experiments were carried out in JET H-mode pulses, where the momentum source was modulated using the Neutral Beam Injection [101]. It was demonstrated that both the amplitude and phase of the modulated toroidal velocity from the experiment are best reproduced when a momentum pinch is taken into account. In this analysis, the residual stress contribution was neglected. This JET pulse was analyzed by QuaLiKiz. The estimated Prandtl number lies within 0.8 and 1.4, close to both GKW and experimental values [101]. The pinch number calculated with QuaLiKiz ranges from -3 to -7 , in good agreement with the experimental values. However a definitive conclusion would require smaller error bars. Moreover some significant contributions to the residual stress reviewed in [81] are not taken into account in local models such as QuaLiKiz.

\section{CONCLUSION AND PERSPECTIVES}

The quasilinear approximation was demonstrated to be valid over a wide range of core tokamak parameters using both nonlinear gyrokinetic simulations and turbulence measurements [17, 43, 52, 59]. However, the construction of the saturated potential through nonlinear mechanisms needs to be further improved by revisiting renormalized quasilinear theory. Indeed for low magnetic shear, $|s|<0.6$, ad-hoc formation had to be used. The QuaLiKiz particle fluxes for strongly inward fluxes do not reproduce well nonlinear fluxes. Recent observation of a quasi-coherent structure in measured frequency spectra linked to unstable TEM [64] is also pointing towards the need of a revisited renormalized quasilinear theory.

Moreover, now that QuaLiKiz can be used in an integrated framework, hence flux driven, its predicted profiles can be 
compared to nonlinear gyrokinetic flux driven codes such as GYSELA [102]. Such investigations should allow exploring further the validity domain of the quasilinear approximation. For example, it would be interesting to explore quantitatively the impact of reported avalanches [103] on the overall transport level and their parametric behavior.

Nonetheless, as demonstrated by TGLF and other quasilinear studies, over a wide range of parameters quasilinear energy, particle and momentum fluxes reproduce well nonlinear gyrokinetic fluxes while gaining a factor $10^{6}$ on the computing time. In the case of QuaLiKiz, the turbulent fluxes for 20 radial positions and 10 wave numbers can be computed in less than 5 minutes. With such a code, 1 to $10 \mathrm{~s}$ of plasma evolution can be modeled within a $24 \mathrm{~h}$ using a very easily accessible number of 30 CPUs. Note that such time scales are similar to TGLF [24]. QuaLiKiz imbedded in CRONOS [37] reproduces well temperature profiles of in JET baseline [38], ohmic L mode temperature in Tore Supra up to $r / a=0.8$ as well as slowly evolving hollow density profiles [39]. The L mode edge issue encounter on DIII-D cases [94, 96, 104] needs to be investigated with QuaLiKiz, as well as current ramp-up modeling which is challenging edge turbulent electron heat transport $[105,106]$. To model high $\beta$ hybrid $\mathrm{H}$ modes[38], QuaLiKiz which is presently electrostatic needs to be extended to included nonlinear electromagnetic stabilization [34]. Accounting for electromagnetic effects and general geometry is expected to slow down QuaLiKiz. It is presently marginally fast enough, therefore an alternative route is being explored to include richer physical effects while speeding up the calculation. This is based on using a Neural Network emulation on a large QuaLiKiz database at first, then a database including results from GKW, GENE, etc. The proof of principle of this idea was successfully demonstrated on 5D QuaLiKiz database with adiabatic electrons [107]. It is presently being extended to kinetic electrons and up to 10D.

Despite various open issues to be addressed, QuaLiKiz is an adequate quasilinear gyrokinetic turbulent transport code for integrated modeling of $\mathrm{H}$ mode baseline and $\mathrm{L}$ mode core. It is very rapid and, if used in stand alone, it can allow for extensive input scans which are essential when comparing the results to experimental fluxes. Indeed fixed gradient codes are highly sensitive to the uncertainties on its inputs such as the normalized temperature gradient for example. When used in a source driven integrated framework such as CRONOS, TGLF or QuaLiKiz model temperatures, densities and rotation profiles of few seconds of plasma. Hence now, two first principle based turbulent transport codes, bridging theory and experiments are available to all interested users. QuaLiKiz will be coupled in the European Transport Solver and JETTO by end of 2015. Documentation on code derivation details are available online [43]. For more information and to download the code, contact the two main authors of this article: clarisse.bourdelle@cea.fr or j.citrin@differ.nl.

\section{Acknowledgments}

The work presented in this article is the result of a collaborative work.

The main author, CB, would like to acknowledge all her coauthors and co-workers over the past 15 years from profile and turbulence measurements, tokamak operation, experimental analysis, gyrokinetic modeling and turbulence theory: C. Angioni, H. Arnichand, J-F Artaud, Y. Baranov, M. Beurskens, P. Beyer, R. Budny, Y. Camenen, J. Candy, C. Challis, L. Chôné, B. Chouli, F. Clairet, W. Dorland, N. Dubuit, R. Dumont, D. Escande, G. Falchetto, C. Fenzi, J. Garcia, T. Gerbeaud, G. Giruzzi, V. Grandgirard, R. Guirlet, O. Gürcan, G. Hammett, P. Hennequin, G.T. Hoang, W. Horton, F. Jenko, E. Joffrin, X. Litaudon, A. Loarte, J. Mailloux, M. Maslov, S. Moradi, M. Ottaviani, T. Parisot, C. Passeron, C. Maggi, F. Militello, M. Romanelli, G. Regnoli, F. Ryter, R. Sabot, Y. Sarazin, A. Smolyakov, G. Staebler, D. Stutman, E. Synakowski, T. Tala, E. Trier, L. Vermare, D. Villegas, R. Waltz, W. Zhong, X.L. Zou.

An additional special thank to G. Staebler for his help in putting up table II.

This work has been carried out within the framework of the EUROfusion Consortium and has received funding from the Euratom research and training programme 2014-2018 under grant agreement No 633053. The views and opinions expressed herein do not necessarily reflect those of the European Commission.
[1] F. Jenko. Computer Physics Communications, 125(1-3):196209, 2000.

[2] A. G. Peeters and D. Strintzi. The effect of a uniform radial electric field on the toroidal ion temperature gradient mode. Physics of Plasmas, 11(8):3748-3751, 2004.

[3] Mike Kotschenreuther, G. Rewoldt, and W.M. Tang. Comparison of initial value and eigenvalue codes for kinetic toroidal plasma instabilities. Computer Physics Communications, 88(2-3):128-140, 1995.

[4] J. Candy and R.E. Waltz. An eulerian gyrokinetic-maxwell solver. Journal of Computational Physics, 186(2):545 - 581, 2003.

[5] V. Grandgirard, M. Brunetti, P. Bertrand, N. Besse, X. Garbet, P. Ghendrih, G. Manfredi, Y. Sarazin, O. Sauter, E. Sonnen-
drÃijcker, J. Vaclavik, and L. Villard. A drift-kinetic semilagrangian $4 \mathrm{~d}$ code for ion turbulence simulation. Journal of Computational Physics, 217(2):395 - 423, 2006.

[6] Y. Idomura, S. Tokuda, and Y. Kishimoto. Global profile effects and structure formations in toroidal electron temperature gradient driven turbulence. Nuclear Fusion, 45(12):15711581, 2005

[7] C. Angioni, A.G. Peeters, X. Garbet, A. Manini, F. Ryter, and ASDEX Upgrade Team. Density response to central electron heating: theoretical investigations and experimental observations in asdex upgrade. Nuclear Fusion, 44(8):827, 2004.

[8] C. Bourdelle, X. Garbet, F. Imbeaux, A. Casati, N. Dubuit, R. Guirlet, and T. Parisot. A new gyrokinetic quasilinear transport model applied to particle transport in tokamak plasmas. 
Physics of Plasmas, 14(11), 2007.

[9] C Angioni, R Dux, E Fable, and a G Peeters. Non-adiabatic passing electron response and outward impurity convection in gyrokinetic calculations of impurity transport in ASDEX Upgrade plasmas. Plasma Physics and Controlled Fusion, 49(12):2027-2043, December 2007.

[10] M. E. Puiatti, M. Valisa, C. Angioni, L. Garzotti, P. Mantica, M. Mattioli, L. Carraro, I. Coffey, C. Sozzi, and JET-EFDA contributors. Analysis of metallic impurity density profiles in low collisionality joint european torus h-mode and l-mode plasmas. Physics of Plasmas (1994-present), 13(4), 2006.

[11] C. Giroud, R. Barnsley, P. Buratti, I.H. Coffey, M. von Hellermann, C. Jupén, K.D. Lawson, A. Meigs, M. O’Mullane, A.D. Whiteford, K.-D. Zastrow, and the JET EFDA contributors. Method for experimental determination of $\mathrm{z}$ dependence of impurity transport on jet. Nuclear Fusion, 47(4):313, 2007.

[12] D. Villegas, R. Guirlet, C. Bourdelle, G. T. Hoang, X. Garbet, and R. Sabot. Experimental electron temperature gradient dependence of heavy impurity transport in fusion devices. Physical Review Letters, 105(3), 2010.

[13] Manas P and Camenen Y et al. 42nd EPS Conference on Controlled Fusion and Plasma Physics, Lisbon, 2015.

[14] C. Angioni, P. Mantica, T. Pütterich, M. Valisa, M. Baruzzo, E.A. Belli, P. Belo, F.J. Casson, C. Challis, P. Drewelow, C. Giroud, N. Hawkes, T.C. Hender, J. Hobirk, T. Koskela, L. Lauro Taroni, C.F. Maggi, J. Mlynar, T. Odstrcil, M.L. Reinke, M. Romanelli, and JET EFDA Contributors. Tungsten transport in jet h-mode plasmas in hybrid scenario, experimental observations and modelling. Nuclear Fusion, 54(8):083028, 2014.

[15] F J Casson, C Angioni, E A Belli, R Bilato, P Mantica, T Odstrcil, T PÃijtterich, M Valisa, L Garzotti, C Giroud, J Hobirk, C F Maggi, J Mlynar, and M L Reinke. Theoretical description of heavy impurity transport and its application to the modelling of tungsten in jet and asdex upgrade. Plasma Physics and Controlled Fusion, 57(1):014031, 2015.

[16] C. Angioni, F. J. Casson, P. Mantica, T. PÃijtterich, M. Valisa, E. A. Belli, R. Bilato, C. Giroud, P. Helander, and JET Contributors. The impact of poloidal asymmetries on tungsten transport in the core of jet h-mode plasmas. Physics of Plasmas, 22(5):-, 2015.

[17] P Cottier, C Bourdelle, Y Camenen, Ö D Gürcan, F J Casson, $\mathrm{X}$ Garbet, $\mathrm{P}$ Hennequin, and $\mathrm{T}$ Tala. Angular momentum transport modeling: achievements of a gyrokinetic quasi-linear approach. Plasma Physics and Controlled Fusion, 56(1):015011, 2014.

[18] C. Angioni, Y. Camenen, F.J. Casson, E. Fable, R.M. McDermott, a.G. Peeters, and J.E. Rice. Off-diagonal particle and toroidal momentum transport: a survey of experimental, theoretical and modelling aspects. Nuclear Fusion, 52(11):114003, November 2012.

[19] H. Weisen, Y. Camenen, A. Salmi, T. W. Versloot, P. C. de Vries, M. Maslov, T. Tala, M. Beurskens, C. Giroud, and JET-EFDA Contributors. Identification of the ubiquitous Coriolis momentum pinch in JET tokamak plasmas. NUCLEAR FUSION, 52(4), APR 2012.

[20] M. Barnes, I. G. Abel, W. Dorland, T. GÃúrler, G. W. Hammett, and F. Jenko. Direct multiscale coupling of a transport code to gyrokinetic turbulence codes. Physics of Plasmas (1994-present), 17(5), 2010.

[21] M. Kotschenreuther, W. Dorland, M. A. Beer, and G. W. Hammett. Quantitative predictions of tokamak energy confinement from first principles simulations with kinetic effects. Physics of Plasmas (1994-present), 2(6):2381-2389, 1995.
[22] A. G. Peeters, Y. Camenen, F. J. Casson, W. A. Hornsby, A. P. Snodin, D. Strintzi, and G. Szepesi. The nonlinear gyrokinetic flux tube code GKW. Computer Physics Communications, 180(12):2650-2672, 2009.

[23] G. M. Staebler, J. E. Kinsey, and R. E. Waltz. Gyro-landau fluid equations for trapped and passing particles. Physics of Plasmas (1994-present), 12(10), 2005.

[24] G. M. Staebler, R. E. Waltz, J. Candy, and J. E. Kinsey. New paradigm for suppression of gyrokinetic turbulence by velocity shear. Phys. Rev. Lett., 110:055003, Jan 2013.

[25] J. Weiland, A.B. Jarmèn, and H. Nordman. Diffusive particle and heat pinch effects in toroidal plasmas. Nuclear Fusion, 29(10):1810, 1989.

[26] Glenn Bateman, Arnold H. Kritz, Jon E. Kinsey, Aaron J. Redd, and Jan Weiland. Predicting temperature and density profiles in tokamaks. Physics of Plasmas (1994-present), 5(5):1793-1799, 1998.

[27] T. Rafiq, A. H. Kritz, V. Tangri, A. Y. Pankin, I. Voitsekhovitch, R. V. Budny, and JET EFDA Contributors. Integrated modeling of temperature profiles in 1-mode tokamak discharges. Physics of Plasmas, 21(12):-, 2014.

[28] RE Waltz, GM Staebler, W Dorland, GW Hammett, M Kotschenreuther, and JA Konings. A gyro-Landau-fluid transport model. Physics of Plasmas, 4(7):2482-2496, JUL 1997.

[29] J. E. Kinsey, G. M. Staebler, and R. E. Waltz. The first transport code simulations using the trapped gyro-landau-fluid model. Physics of Plasmas (1994-present), 15(5), 2008.

[30] F. Imbeaux and Y. Peysson. Ray-tracing and fokker-planck modelling of the effect of plasma current on the propagation and absorption of lower hybrid waves. Plasma Physics and Controlled Fusion, 47(11):2041-2065, 2005.

[31] T Tala, F Imbeaux, V.V Parail, C Bourdelle, G Corrigan, X Garbet, D.J Heading, X Litaudon, P.I Strand, J Weiland, and Jet-Efda Contributors. Fully predictive time-dependent transport simulations of ITB plasmas in JET, JT-60U and DIII-D. Nuclear Fusion, 46(5):548-561, May 2006.

[32] J.E. Kinsey, G.M. Staebler, J. Candy, R.E. Waltz, and R.V. Budny. Iter predictions using the gyro verified and experimentally validated trapped gyro-landau fluid transport model. Nuclear Fusion, 51(8):083001, 2011.

[33] C. Holland, J.E. Kinsey, J.C. DeBoo, K.H. Burrell, T.C. Luce, S.P. Smith, C.C. Petty, A.E. White, T.L. Rhodes, L. Schmitz, E.J. Doyle, J.C. Hillesheim, G.R. McKee, Z. Yan, G. Wang, L. Zeng, B.A. Grierson, A. Marinoni, P. Mantica, P.B. Snyder, R.E. Waltz, G.M. Staebler, and J. Candy. Validation studies of gyrofluid and gyrokinetic predictions of transport and turbulence stiffness using the DIII-D tokamak. Nuclear Fusion, 53(8):083027, August 2013.

[34] J. Citrin, F. Jenko, P. Mantica, D. Told, C. Bourdelle, J. Garcia, J. W. Haverkort, G. M. D. Hogeweij, T. Johnson, and M. J. Pueschel. Nonlinear Stabilization of Tokamak Microturbulence by Fast Ions. Physical Review Letters, 111(15):155001, October 2013.

[35] J. Citrin and C. Bourdelle et al. ITPA Transport and Confinement, October 2014, Cadarache, 2014.

[36] Ö. D. Gürcan. Numerical computation of the modified plasma dispersion function with curvature. Journal of Computational Physics, 269(0):156 - 167, 2014.

[37] J. F. Artaud, V. Basiuk, F. Imbeaux, M. Schneider, J. Garcia, G. Giruzzi, P. Huynh, T. Aniel, F. Albajar, J. M. Ane, A. Becoulet, C. Bourdelle, A. Casati, L. Colas, J. Decker, R. Dumont, L. G. Eriksson, X. Garbet, R. Guirlet, P. Hertout, G. T. Hoang, W. Houlberg, G. Huysmans, E. Joffrin, S. H. Kim, 
F. Koechl, J. Lister, X. Litaudon, P. Maget, R. Masset, B. Pegourie, Y. Peysson, P. Thomas, E. Tsitroneand, and F. Turco. The cronos suite of codes for integrated tokamak modelling. Nuclear Fusion, 50(4), 2010.

[38] B Baiocchi, J Garcia, M Beurskens, C Bourdelle, F Crisanti, C Giroud, J Hobirk, F Imbeaux, I Nunes, JET Contributors, and EU-ITM ITER Scenario Modelling group. Turbulent transport analysis of jet h-mode and hybrid plasmas using qualikiz and trapped gyro landau fluid. Plasma Physics and Controlled Fusion, 57(3):035003, 2015.

[39] Baiocchi B., Bourdelle C., Angioni C., Imbeaux F., Loarte A., Maslov M., and JET EFDA contributors. Transport analysis and modelling of the evolution of hollow density profiles plasmas in jet and implication for iter. To be published in Nuclear Fusion, 2015.

[40] R. L. Miller, M. S. Chu, J. M. Greene, Y. R. Lin-Liu, and R. E. Waltz. Noncircular, finite aspect ratio, local equilibrium model. Physics of Plasmas (1994-present), 5(4):973978, 1998.

[41] T. Gerbaud, F. Clairet, R. Sabot, A. Sirinelli, S. Heuraux, G. Leclert, and L. Vermare. Comparison of density fluctuation measurements between o-mode and x-mode reflectometry on tore supra. Review of Scientific Instruments, 77(10):10E928, 2006.

[42] C. Bourdelle, X. Garbet, G.T. Hoang, J. Ongena, and R.V. Budny. Nuclear Fusion, 42(7):892, 2002.

[43] C. Bourdelle. Turbulent transport in tokamak plasmas: bridging theory and experiment. Habilitation à Diriger des Recherches, Aix-Marseille University, 2015.

[44] X. Garbet. Instabilités, turbulence et transport dans un plasma magnétisé. Thèse d'habilitation à diriger des recherches, Université de Provence, Aix-Marseille I, 2001.

[45] A. J. Brizard and T. S. Hahm. Foundations of nonlinear gyrokinetic theory. Review of Modern Physics, 79(2), 2007.

[46] J. W. Connor, R. J. Hastie, and J. B. Taylor. High mode number stability of an axisymmetric toroidal plasma. Proceedings of the Royal Society of London. A. Mathematical and Physical Sciences, 365(1720):1-17, 1979.

[47] M.N. Rosenbluth J. Candy, R. Waltz. Physics of Plasmas, 11(5):1879, 2004.

[48] John Wesson. Tokamaks (second edition). Oxford Engineering Science Series, Clarendon Press, (ISBN 0-19-856293-4), 1997.

[49] V. D. Shafranov. Plasma Equilibrium in a Magnetic Field. Reviews of Plasma Physics, 2:103, 1966.

[50] M. Romanelli, G. T. Hoang, C. Bourdelle, C. Gormezano, E. Giovannozzi, M. Leigheb, M. Marinucci, D. Marocco, C. Mazzotta, L. Panaccione, V. Pericoli, G. Regnoli, O. Tudisco, and F. T. U. Team. Parametric dependence of turbulent particle transport in high density electron heated ftu plasmas. Plasma Physics and Controlled Fusion, 49(6):935-946, 2007.

[51] G. Rewoldt, W. M. Tang, and M. S. Chance. Electromagnetic kinetic toroidal eigenmodes for general magnetohydrodynamic equilibria. Physics of Fluids (1958-1988), 25(3):480-490, 1982.

[52] J. Citrin, C. Bourdelle, P. Cottier, D. F. Escande, Oe D. Guercan, D. R. Hatch, G. M. D. Hogeweij, F. Jenko, and M. J. Pueschel. Quasilinear transport modelling at low magnetic shear. Physics of Plasmas, 19(6), JUN 2012.

[53] B Davies. Locating the zeros of an analytic function. Journal of Computational Physics, 66(1):36 - 49, 1986.

[54] M. Romanelli, C. Bourdelle, and W. Dorland. Effects of high density peaking and high collisionality on the stabilization of the electrostatic turbulence in the frascati tokamak upgrade.
Physics of Plasmas, 11(8):3845-3853, 2004.

[55] John A. Krommes. Fundamental statistical descriptions of plasma turbulence in magnetic fields. Physics Reports, 360(1 - 4): 1 - 352, 2002.

[56] Ryogo Kubo. Stochastic liouville equations. Journal of Mathematical Physics, 4(2):174-183, 1963.

[57] John A. Krommes. Fundamental statistical descriptions of plasma turbulence in magnetic fields. Physics Reports, 360(1 - 4): 1 - 352, 2002.

[58] Y. Lin, J. Rice, S. Wukitch, M. Greenwald, a. Hubbard, a. Ince-Cushman, L. Lin, M. Porkolab, M. Reinke, and N. Tsujii. Observation of Ion-Cyclotron-Frequency ModeConversion Flow Drive in Tokamak Plasmas. Physical Review Letters, 101(23):235002, December 2008.

[59] A. Casati, C. Bourdelle, X. Garbet, F. Imbeaux, J. Candy, F. Clairet, G. Dif-Pradalier, G. Falchetto, T. Gerbaud, V. Grandgirard, Oe D. Guercan, P. Hennequin, J. Kinsey, M. Ottaviani, R. Sabot, Y. Sarazin, L. Vermare, and R. E. Waltz. Validating a quasi-linear transport model versus nonlinear simulations. Nuclear Fusion, 49(8), 2009.

[60] T. H. Dupree. A perturbation theory for strong plasma turbulence. Physics of Fluids (1958-1988), 9(9):1773-1782, 1966.

[61] A. Casati. A quasi-linear gyrokinetic transport model for tokamak plasmas. PhD thesis, Université de Provence, AixMarseille I, 2009.

[62] A. Casati, T. Gerbaud, P. Hennequin, C. Bourdelle, J. Candy, F. Clairet, X. Garbet, V. Grandgirard, Oe D. Guercan, S. Heuraux, G. T. Hoang, C. Honore, F. Imbeaux, R. Sabot, Y. Sarazin, L. Vermare, and R. E. Waltz. Turbulence in the tore supra tokamak: Measurements and validation of nonlinear simulations. Physical Review Letters, 102(16), 2009.

[63] D. R. Hatch, F. Jenko, A. Bañón Navarro, and V. Bratanov. Transition between saturation regimes of gyrokinetic turbulence. Phys. Rev. Lett., 111:175001, Oct 2013.

[64] H. Arnichand, R. Sabot, S. Hacquin, A. KrÃd'mer-Flecken, X. Garbet, J. Citrin, C. Bourdelle, G. Hornung, J. Bernardo, C. Bottereau, F. Clairet, G. Falchetto, and J.C. Giacalone. Quasi-coherent modes and electron-driven turbulence. $\mathrm{Nu}$ clear Fusion, 54(12):123017, 2014.

[65] J. Citrin, H. Arnichand, R. Sabot, S. Hacquin, X. Garbet, and C. Bourdelle. Quasi-coherent modes and electron-driven turbulence. TTF Culham, 2014.

[66] T. Dannert and F. Jenko. Gyrokinetic simulation of collisionless trapped-electron mode turbulence. Physics of Plasmas, 12(7):072309, 2005.

[67] R. E. Waltz, a. Casati, and G. M. Staebler. Gyrokinetic simulation tests of quasilinear and tracer transport. Physics of Plasmas, 16(7):072303, 2009.

[68] D. R. Hatch, P. W. Terry, W. M. Nevins, and W. Dorland. Role of stable eigenmodes in gyrokinetic models of ion temperature gradient turbulence. Physics of Plasmas, 16(2):022311, 2009.

[69] C.P. Ritz, D.L. Brower, T.L. Rhodes, R.D. Bengtson, S.J. Levinson, N.C. Luhmann Jr., W.A. Peebles, and E.J. Powers. Characterization of tokamak edge turbulence by farinfrared laser scattering and langmuir probes. Nuclear Fusion, 27(7):1125, 1987.

[70] P Devynck, X Garbet, C Laviron, J Payan, S K Saha, F Gervais, $\mathrm{P}$ Hennequin, A Quemeneur, and A Truc. Localized measurements of turbulence in the tore supra tokamak. Plasma Physics and Controlled Fusion, 35(1):63, 1993.

[71] P Hennequin, R Sabot, C Honoré, G T Hoang, X Garbet, A Truc, C Fenzi, and A Quéméneur. Scaling laws of density fluctuations at high-k on tore supra. Plasma Physics and Controlled Fusion, 46(12B):B121-B133, 2004. 
[72] Ö. D. Gürcan and R. Grappin. Anisotropic shell model of turbulence. Phys. Rev. E, 84:066308, Dec 2011.

[73] B. B. Kadomtsev, M. N. Rosenbluth, and W. B. Thompson. Plasma physics: Lectures presented at the seminar on plasma physics organized by and held at the international centre for theoretical physics, trieste, october 1964. International Atomic Energy Agency: Vienna, 1965.

[74] Kimitaka Itoh and Sanae-I Itoh. The role of the electric field in confinement. Plasma Physics and Controlled Fusion, 38(1):1, 1996.

[75] G.R. McKee, C.C. Petty, R.E. Waltz, C. Fenzi, R.J. Fonck, J.E. Kinsey, T.C. Luce, K.H. Burrell, D.R. Baker, E.J. Doyle, X. Garbet, R.A. Moyer, C.L. Rettig, T.L. Rhodes, D.W. Ross, G.M. Staebler, R. Sydora, and M.R. Wade. Non-dimensional scaling of turbulence characteristics and turbulent diffusivity. Nuclear Fusion, 41(9), 2001.

[76] A Hirose, S Livingstone, and A.K Singh. On q dependence of thermal transport in tokamaks. Nuclear Fusion, 45(12):16281633, December 2005.

[77] F Jenko, T Dannert, and C Angioni. Heat and particle transport in a tokamak: advances in nonlinear gyrokinetics. Plasma Physics and Controlled Fusion, 47(12B):B195-B206, December 2005.

[78] G. L. Falchetto and M. Ottaviani. Effect of collisional zonalflow damping on flux-driven turbulent transport. Physical Review Letters, 92(2):025002, 2004.

[79] A. M. Dimits, G. Bateman, M. A. Beer, B. I. Cohen, W. Dorland, G. W. Hammett, C. Kim, J. E. Kinsey, M. Kotschenreuther, A. H. Kritz, L. L. Lao, J. Mandrekas, W. M. Nevins, S. E. Parker, A. J. Redd, D. E. Shumaker, R. Sydora, and J. Weiland. Comparisons and physics basis of tokamak transport models and turbulence simulations. Physics of Plasmas, 7(3):969-983, 2000.

[80] D.R. Mikkelsen and W. Dorland. Phys. Rev. Lett., 101:135003, 2008.

[81] R. E. Waltz, G. M. Staebler, and W. M. Solomon. Gyrokinetic simulation of momentum transport with residual stress from diamagnetic level velocity shears. Physics of Plasmas, 18(4):042504, 2011.

[82] T. S. Hahm, P. H. Diamond, O. D. Gurcan, and G. Rewoldt. Nonlinear gyrokinetic theory of toroidal momentum pinch. Physics of Plasmas, 14(7):072302, 2007.

[83] A.G. Peeters, C. Angioni, A. Bortolon, Y. Camenen, F.J. Casson, B. Duval, L. Fiederspiel, W.A. Hornsby, Y. Idomura, T. Hein, N. Kluy, P. Mantica, F.I. Parra, A.P. Snodin, G. Szepesi, D. Strintzi, T. Tala, G. Tardini, P. de Vries, and J. Weiland. Overview of toroidal momentum transport. $\mathrm{Nu}$ clear Fusion, 51(9):094027, September 2011.

[84] A. G. Peeters and C. Angioni. Linear gyrokinetic calculations of toroidal momentum transport in a tokamak due to the ion temperature gradient mode. Physics of Plasmas, 12(7):072515, 2005.

[85] C Bourdelle. Turbulent particle transport in magnetized fusion plasma. Plasma Physics and Controlled Fusion, 47(5A, SI):A317-A326, 2005.

[86] C. Angioni, J. Candy, E. Fable, M. Maslov, a. G. Peeters, R. E. Waltz, and H. Weisen. Particle pinch and collisionality in gyrokinetic simulations of tokamak plasma turbulence. Physics of Plasmas, 16(6):060702, 2009.

[87] A. Loarte, M.J. Leyland, J.A. Mier, M.N.A. Beurskens, I. Nunes, V. Parail, P.J. Lomas, G.R. Saibene, R.I.A. Sartori, L. Frassinetti, and JET EFDA Contributors. Plasma density and temperature evolution following the h-mode transition at jet and implications for iter. Nuclear Fusion, 53(8):083031,
2013.

[88] R. Guirlet, A. Sirinelli, T. Parisot, R. Sabot, J. F. Artaud, C. Bourdelle, X. Garbet, P. Hennequin, G. T. Hoang, F. Imbeaux, J. L. Ségui, D. Mazon, and D. Villegas. Particle transport in low core turbulence tore-supra plasmas. Nuclear Fusion, 50(9), 2010.

[89] T. Parisot, R. Guirlet, C. Bourdelle, X. Garbet, N. Dubuit, F. Imbeaux, and P. R. Thomas. Experimental impurity transport and theoretical interpretation in a tore supra lower-hybrid heated plasma. Plasma Physics and Controlled Fusion, 50(5), 2008.

[90] R Dux, R Neu, a G Peeters, G Pereverzev, a M ck, F Ryter, J Stober, and Asdex Upgrade Team. Influence of the heating profile on impurity transport in ASDEX Upgrade. Plasma Physics and Controlled Fusion, 45(9):1815-1825, September 2003.

[91] C. Angioni. Theoretical and experimental research on particle and impurity transport in the core of tokamak plasmas. Thèse d'habilitation à diriger des recherches, Université de Provence, Aix-Marseille I, 2010.

[92] H Nordman, A Skyman, P Strand, C Giroud, F Jenko, F Merz, V Naulin, T Tala, and the JET-EFDA Contributors. Fluid and gyrokinetic simulaftions of impurity transport at jet. Plasma Physics and Controlled Fusion, 53(10):105005, 2011.

[93] C. Angioni and A. G. Peeters. Direction of impurity pinch and auxiliary heating in tokamak plasmas. Phys. Rev. Lett., 96:095003, Mar 2006.

[94] A. E. White, W. A. Peebles, T. L. Rhodes, C. H. Holland, G. Wang, L. Schmitz, T. A. Carter, J. C. Hillesheim, E. J. Doyle, L. Zeng, G. R. McKee, G. M. Staebler, R. E. Waltz, J. C. DeBoo, C. C. Petty, and K. H. Burrell. Measurements of the cross-phase angle between density and electron temperature fluctuations and comparison with gyrokinetic simulations. Physics of Plasmas, 17(5), 2010.

[95] C. Holland, L. Schmitz, T. L. Rhodes, W. A. Peebles, J. C. Hillesheim, G. Wang, L. Zeng, E. J. Doyle, S. P. Smith, R. Prater, K. H. Burrell, J. Candy, R. E. Waltz, J. E. Kinsey, G. M. Staebler, J. C. DeBoo, C. C. Petty, G. R. McKee, Z. Yan, and A. E. White. Advances in validating gyrokinetic turbulence models against 1 - and h-mode plasmas. Physics of Plasmas, 18(5), 2011.

[96] J. E. Kinsey, G. M. Staebler, J. Candy, C. C. Petty, T. L. Rhodes, and R. E. Waltz. Predictions of the near edge transport shortfall in diii-d l-mode plasmas using the trapped gyrolandau-fluid model. Physics of Plasmas, 22(1):-, 2015.

[97] C Bourdelle, X Garbet, R Singh, and L Schmitz. Plasma Physics and Controlled Fusion, 54(11):115003, 2012.

[98] Camenen Y et al. 42nd EPS Conference on Controlled Fusion and Plasma Physics, Lisbon, 2015.

[99] Ö. D. Gürcan, L. Vermare, P. Hennequin, V. Berionni, P.H. Diamond, G. Dif-Pradalier, X. Garbet, P. Ghendrih, V. Grandgirard, C.J. McDevitt, P. Morel, Y. Sarazin, a. Storelli, and C. Bourdelle. Structure of nonlocality of plasma turbulence. Nuclear Fusion, 53(7):073029, July 2013.

[100] N T Howard, C Holland, A E White, M Greenwald, and $\mathrm{J}$ Candy. Fidelity of reduced and realistic electron mass ratio multi-scale gyrokinetic simulations of tokamak discharges. Plasma Physics and Controlled Fusion, 57(6):065009, 2015.

[101] T. Tala, K.-D. Zastrow, J. Ferreira, P. Mantica, V. Naulin, a. Peeters, G. Tardini, M. Brix, G. Corrigan, C. Giroud, and D. Strintzi. Evidence of Inward Toroidal Momentum Convection in the JET Tokamak. Physical Review Letters, 102(7):075001, February 2009.

[102] Y Sarazin, a Strugarek, G Dif-Pradalier, J Abiteboul, S All- 
frey, X Garbet, Ph Ghendrih, V Grandgirard, and G Latu. Flux-driven gyrokinetic simulations of ion turbulent transport at low magnetic shear. Journal of Physics: Conference Series, 260:012017, November 2010.

[103] G. Dif-Pradalier, G. Hornung, Ph. Ghendrih, Y. Sarazin, F. Clairet, L. Vermare, P. H. Diamond, J. Abiteboul, T. CartierMichaud, C. Ehrlacher, D. Estève, X. Garbet, V. Grandgirard, Ö. D. Gürcan, P. Hennequin, Y. Kosuga, G. Latu, P. Maget, P. Morel, C. Norscini, R. Sabot, and A. Storelli. Finding the elusive $\mathbf{E} \times \mathbf{B}$ staircase in magnetized plasmas. Phys. Rev. Lett., 114:085004, Feb 2015.

[104] T. GÃuurler, A. E. White, D. Told, F. Jenko, C. Holland, and T. L. Rhodes. A flux-matched gyrokinetic analysis of diii-d 1-mode turbulence. Physics of Plasmas, 21(12):-, 2014.

[105] I. Voitsekhovitch, A. C. C. Sips, B. Alper, M. Beurskens,
I. Coffey, J. Conboy, T. Gerbaud, C. Giroud, T. Johnson, F. Koechl, E. de la Luna, D. C. McDonald, I. Pavlenko, G. V. Pereverzev, S. Popovichev, A. N. Saveliev, G. Sergienko, S. Sharapov, M. Stamp, and Jet-Efda Contributors. Modelling of the jet current ramp-up experiments and projection to iter. Plasma Physics and Controlled Fusion, 52(10), 2010.

[106] E. Fable, C. Angioni, R. Fischer, B. Geiger, R.M. McDermott, G.V. Pereverzev, T. Puetterich, F. Ryter, B. Scott, G. Tardini, E. Viezzer, and the ASDEX Upgrade Team. Progress in characterization and modelling of the current ramp-up phase of asdex upgrade discharges. Nuclear Fusion, 52(6):063017, 2012.

[107] J. Citrin, S. Breton, F. Felici, and F. Imbeaux et al. Real-time capable first principle based modelling of tokamak turbulent transport. Submitted to Nuclear Fusion, 2015. 